\title{
Flujos de biomasa y estructura de un ecosistema de surgencia tropical en La Guajira, Caribe colombiano
}

\author{
Maria Isabel Criales-Hernández ${ }^{1 *}$, Camilo B. García ${ }^{2} \&$ Matthias Wolff ${ }^{3}$ \\ 1 Universidad del Magdalena, AA.1690 Santa Marta, Colombia; micriales@imarpe.gob.pe \\ 2 Universidad Nacional de Colombia, Departamento de Biología, CECIMAR/INVEMAR, AA 1016 Santa Marta, \\ Colombia; cgarcia@invemar.org.co \\ 3 ZMT (Center for Tropical Marine Ecology), Fahrenheistrasse 6, D-28359 Bremen, Alemania; mwolff@uni-bremen.de \\ * Dirección actual: Instituto del Mar de Perú (IMARPE), Área de Evaluación y Producción Secundaria, Esquina de \\ Gamarra y General Valle S/N Chuchito Callao, Perú, Tel: (51)1 4296069.
}

Recibido 18-III-2004. Corregido 27-IX-2005. Aceptado 12-V-2006.

\begin{abstract}
Biomass flow and structure of a tropical upwelling ecosystem in La Guajira, Colombian Caribbean. La Guajira is an exploited tropical upwelling ecosystem in the Colombian Caribbean coast. A trophic model of 27 functional groups was constructed using the ECOPATH 5.0 Beta software to integrate the available information on the ecosystem. The model allowed a comparison with other trophic flow models of upwelling ecosystems. Total system biomass ( $68 \mathrm{t} / \mathrm{km}^{2} /$ year), net system production ( $1248.5 \mathrm{t} / \mathrm{km}^{2} /$ year), and total system throughput (3 $275 \mathrm{t} / \mathrm{km}^{2} /$ year) make La Guajira moderate when compared with other systems. The largest amount of energy throughput is achieved from trophic level I to II (68.93\%), although an important proportion of the total flow originates from detritus (32\%). The production/respiration ratio exceeds 1 , suggesting that La Guajira is an immature ecosystem and is in development, as determined by its low ascendency $(33.7 \%)$ and high development capacity (66.3\%), similar to other upwellings that have values of ascendency between 20 $\%$ and $35 \%$. Although the basic input data were good and covered 1995 to 2000, appropriate information is still not available on some trophic groups such as biomass (for phytoplankton, invertebrates, catfishes and pelagic predator fishes), secondary production data (invertebrates, pelagic predator fishes, and small pelagic fishes), and seabird and mammal populations, which are top trophic levels and an essential part of upwelling ecosystems. Rev. Biol. Trop. 54 (4): 1257-1282. Epub 2006 Dec. 15.
\end{abstract}

Key words: trophic relationships, upwelling ecosystem, Ecopath, Colombia, Caribbean Sea.

Una de las aproximaciones al estudio de los ecosistemas ha sido la valoración de los aspectos funcionales, tales como flujos de materia o energía y ciclo de nutrientes (O’Neill et al. 1986). En el trópico estas aproximaciones involucran necesariamente la agregación de las especies en grupos funcionales debido al elevado número de especies que coexisten en el ecosistema, más de 900 spp en el sistema de surgencia de La Guajira (Navas et al. 2002), por ejemplo. En este tipo de investigaciones se debe tener en cuenta que las interacciones entre las especies y la estructura de las comunidades pueden ser significativamente influenciadas por los cambios experimentados en los regímenes de capturas, lo que produce alteraciones en formas complejas, en respuesta a las perturbaciones ocasionadas por la extracción de los recursos. Así, los ecosistemas se desarrollan dentro de las restricciones impuestas por la productividad primaria, la eficiencia ecotrófica y factores tales como la estabilidad, elasticidad y persistencia del ecosistema (Murawski 1991).

En el presente estudio el área involucrada comprendió un total de $4220.66 \mathrm{~km}^{2}$ entre Riohacha y Punta Gallinas (Fig. 1), localizada en el norte del continente sur americano y 


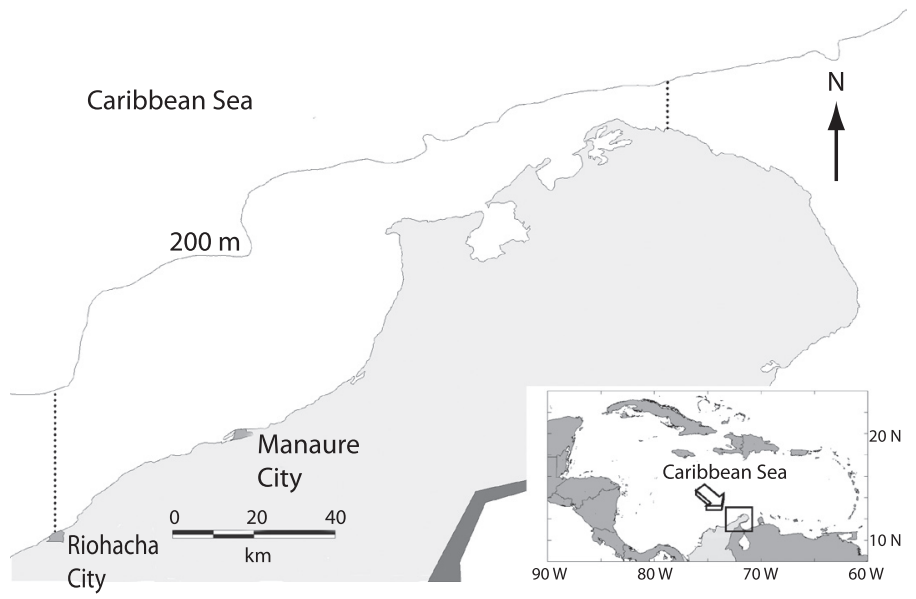

Fig. 1. Ecosistema de surgencia tropical de La Guajira, Caribe colombiano. El área comprende $4220 \mathrm{~km}^{2}$ de plataforma continental detallado por las lineas.

Fig. 1. Upwelling ecosystem off northern Colombia, Caribbean Sea. Study area comprises $4220 \mathrm{~km}^{2}$ of continental shelf indicated by the segmented lines. son uno de los principales productos que se extraen, llegando a constituir casi el $80 \%$ de la captura total de la flota artesanal, sobre todo de especies como Thunnus albacares, T. atlanticus, Scomber japonicus, Euthynnus alleteratus, Decapterus macarellus, Opisthonema oglinum y Sardinella aurita, entre otros (Correa y Manjarrés 2004).

El presente trabajo totalizó la experiencia investigativa de 1995 al 2000 en el área de estudio y a partir de allí se formuló un modelo de funcionamiento del ecosistema de surgencia tropical en La Guajira, Caribe colombiano.

donde se desarrolla una surgencia tropical. La surgencia que se presenta es costera, moderada y es ocasionada por la disposición de la costa irregular en dirección noreste-sureste, la incidencia paralela de los vientos alisios y una plataforma submarina de aguas someras, lo que ocasiona una fuerte divergencia en el transporte de Ekman (Cabrera y Donoso 1993, Andrade 2000). La surgencia se presenta todo el año y es modulada estacionalmente por la variación en la intensidad de los vientos alisios (Andrade 2000). En cuanto a las características del afloramiento, se ha determinado que corresponde a la masa de agua Subtropical Subsuperficial, con temperaturas superficiales entre $22.6{ }^{\circ} \mathrm{C}$ $25.8{ }^{\circ} \mathrm{C}$, que pueden descender hasta $19{ }^{\circ} \mathrm{C}$ en los focos de surgencia activa, y salinidades altas (36.5-37.2 \%o) (Corredor 1979, Cabrera y Donoso 1993). El enriquecimiento biológico se observa particularmente durante la época de intenso afloramiento cuando los pequeños pelágicos se acercan a la costa en aguas someras, especialmente frente a Riohacha (Fig. 1). Esas acumulaciones incluyen especies como Selar crumenophthalmus, Opisthonema oglinum, Decapterus spp. y Sardinella spp. y las biomasas pueden alcanzar $5293 \mathrm{t} / \mathrm{km}^{2}$ (Manjarrés et al. 1997). Los recursos pelágicos
El desarrollar un modelo con Ecopath permite presentar estimaciones biológico pesqueras, describir los flujos de materia y energía del ecosistema, y comparar estos resultados con otros ecosistemas tropicales y de surgencia tropical. Este trabajo resulta útil además como una base para futuras comparaciones de los resultados y valorar posibles consecuencias del cambio en la estructura del ecosistema como producto de escenarios de manejo de las pesquerías.

\section{MATERIALES Y MÉTODOS}

Para la construcción del modelo en el ecosistema de La Guajira, se utilizó el programa Ecopath 5.0 Beta (Christensen et al. 2000), a partir de un modelo de balance de biomasa (en estado de equilibrio) en compartimientos tróficos, según Polovina y Ow (1983). El modelo Ecopath se propone encontrar valores para el consumo de alimento y biomasa de cada grupo definido de modo que el ecosistema se encuentre en balance. Es decir, que para cada caja $i$ (grupo $i$ en el sistema) se cumpla la ecuación básica:

Producción de $i$ - Depredación sobre $i-$ Otras pérdidas de $i-$ Exportación de $i=0$ 
En términos de la formulación matemática del Ecopath la ecuación anterior se expresa como:

$$
B_{i} * P / B_{i} * E E_{i}-\sum B_{j} * Q / B_{j} * D C_{j i}-E X_{i}=0
$$

Donde $B_{i}$ es la biomasa del grupo (i), $P / B_{i}$ es la razón producción a biomasa de $i$, que es igual al coeficiente de mortalidad total $(\mathrm{Z})$ bajo condiciones de estado de equilibrio, $E E_{i}$ es la eficiencia ecotrófica de $i$, que es la parte de la producción que es tomada por depredación, capturas o exportaciones a otros sistemas, $B_{j}$ es la biomasa del depredador $j, Q / B_{j}$ es la razón consumo/biomasa del depredador $j, D C j i$ es la fracción de presa $i$ en la dieta del depredador $\mathrm{j}$ y $E X_{i}$ es la exportación de $i$, que es la suma de las capturas de la pesquería, más la emigración a ecosistemas adyacentes.

Estructura del Modelo: Para el ecosistema de La Guajira se definieron 27 grupos funcionales (Cuadros 1 y 2). En la definición de los grupos funcionales, se tuvieron en cuenta aspectos de la historia natural de las especies como la tasa de consumo (Q/B), la talla, el hábito (demersal o pelágico), la similitud en la dieta y la abundancia relativa (Cuadros 1 y 2). Las especies más importantes comercialmente también fueron incluidas como grupos funcionales (Cuadros 1 y 2).

Datos de entrada: Los datos de entrada del modelo provienen de diferentes fuentes y de las propias estimaciones efectuadas, las cuales se resumen en los Cuadro 1. Cuando se desconocía una estimación del grupo se utilizaron valores Eficiencia Ecotrófica (EE), que corresponden a la fracción de la producción que es usada en el sistema y que puede variar entre 0 y 1 , se espera que se aproxime más a uno cuando el grupo presenta una alta presión por depredación, este dato incluido en algunos casos proviene de grupos similares y modelos informados para el área del Caribe, lo que permite la estimación automática del valor faltante (Christensen et al. 2000). Los datos de entrada y los parámetros estimados después de balancear el modelo y la matriz de dietas se resumen en los Cuadros 2 y 3 .

Balance del modelo: Los datos de biomasa y captura fueron estandarizados a $\mathrm{t} / \mathrm{km}^{2}$. La estrategia seguida para balancear el modelo es obtener valores de Eficiencias Ecotróficas (EE) < 1(tipo de letra) (Polovina 1984a, b) y para el Coeficiente de Conversión Bruta (GE), entre 0.1 y 0.3 , dado que el consumo de la mayoría de los grupos es de tres a diez veces más alto que la producción (Christensen y Pauly 1992). Para esto, la matriz de dietas, la biomasa y el $\mathrm{P} / \mathrm{B}$ fueron gradualmente ajustados hasta obtener un modelo balanceado. En los grupos funcionales ícticos las modificaciones de la biomasa no excedieron el error estándar encontrado en las respectivas estimaciones de cada grupo. En los grupos funcionales de los invertebrados, las modificaciones efectuadas fueron de hasta un $94.6 \%$ (por ejemplo miscelánea de invertebrados herbívoros, miscelánea de invertebrados carnívoros); para efectuar los cambios en estos grupos se tuvo como referencia los valores de densidades promedio encontrados en otros sitios del Caribe. Se hicieron modificaciones en la matriz de dietas, cambiando las fracciones de las presas en los grupos donde se presentaba canibalismo alto como en las correas (Trichiurus lepturus) y anguiliformes, cangrejos y otros crustáceos. Seguidamente se analizaron las tendencias de algunos índices para probar la consistencia del modelo (por ejemplo, la razón respiración/biomasa debe ser más alta en grupos activos que en grupos pasivos).

Después de balancear el modelo, se analizaron algunos atributos de la red para describir las propiedades del ecosistema (Ulanowicz 1986), y algunos índices de los flujos referidos a los atributos de madurez del ecosistema (Odum 1969). Los grupos funcionales del ecosistema pueden ser agregados para representar 
产

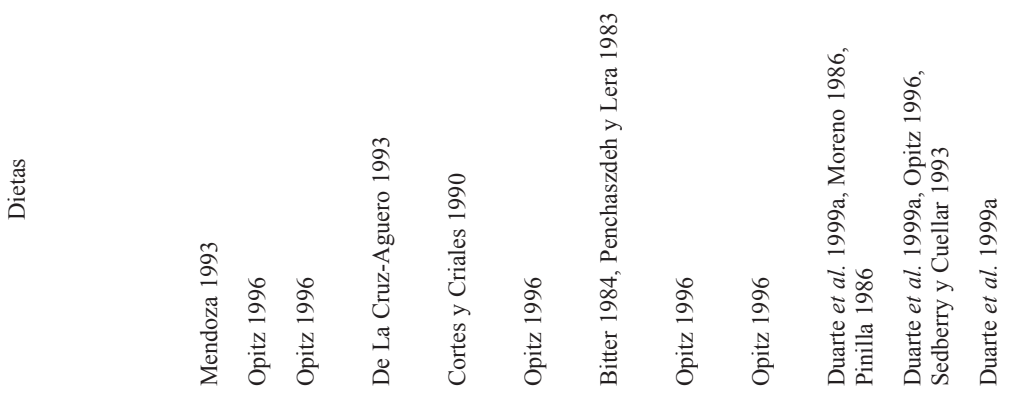

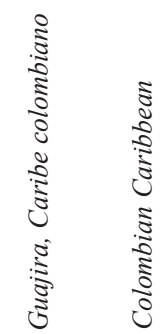

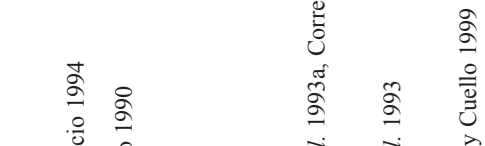

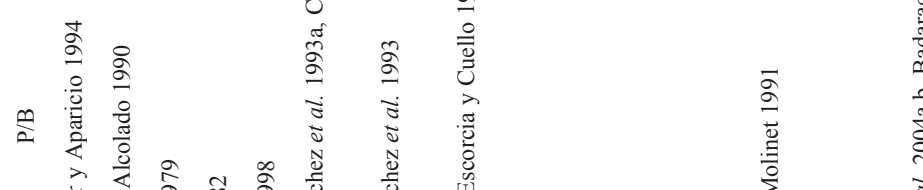

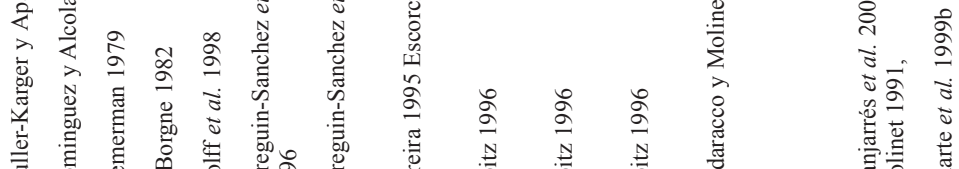

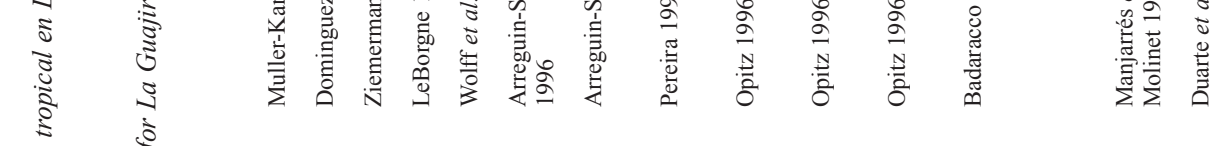

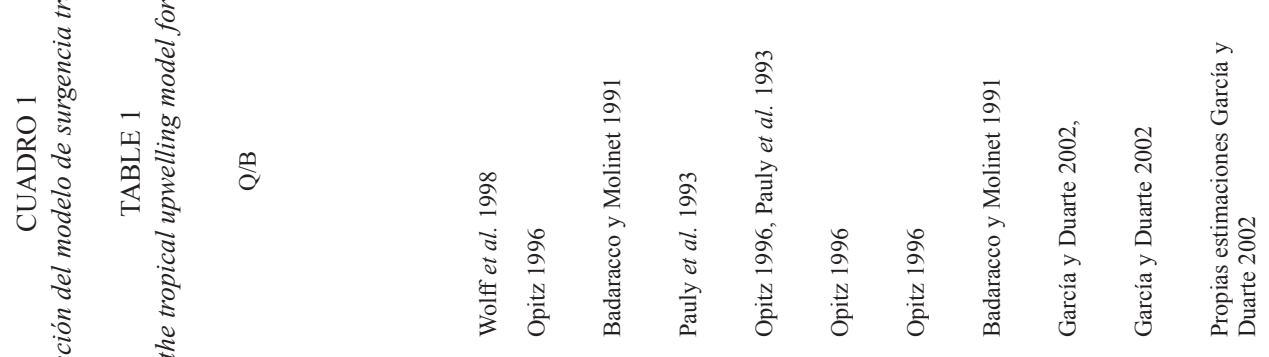<smiles>C1CC2CCCC2C1</smiles>

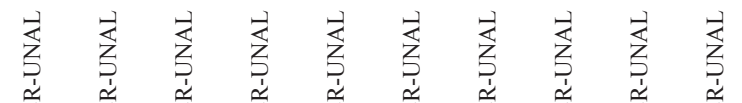

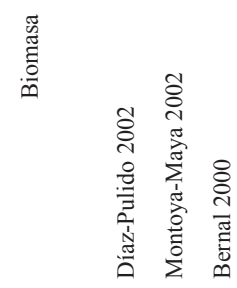

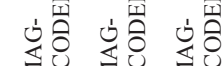

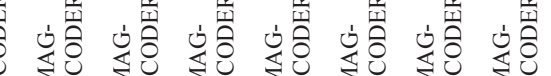

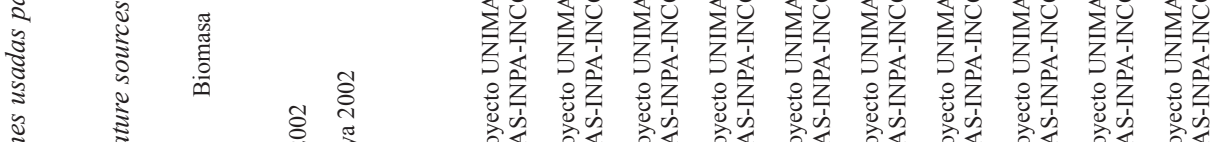

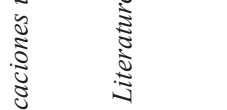

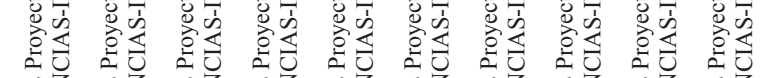

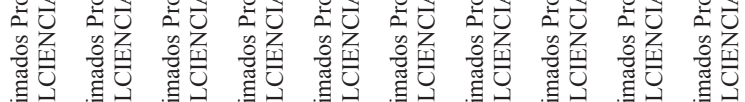

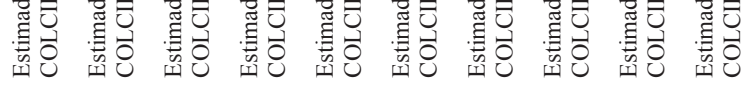

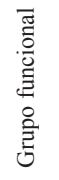

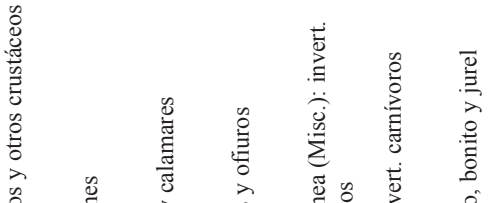

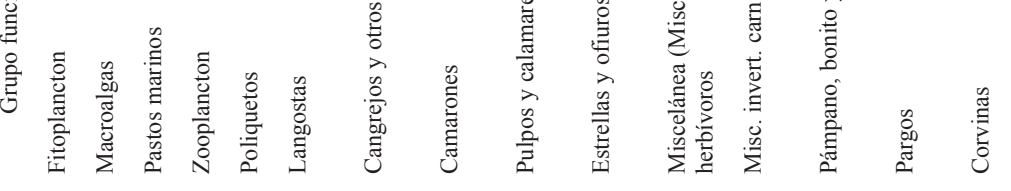




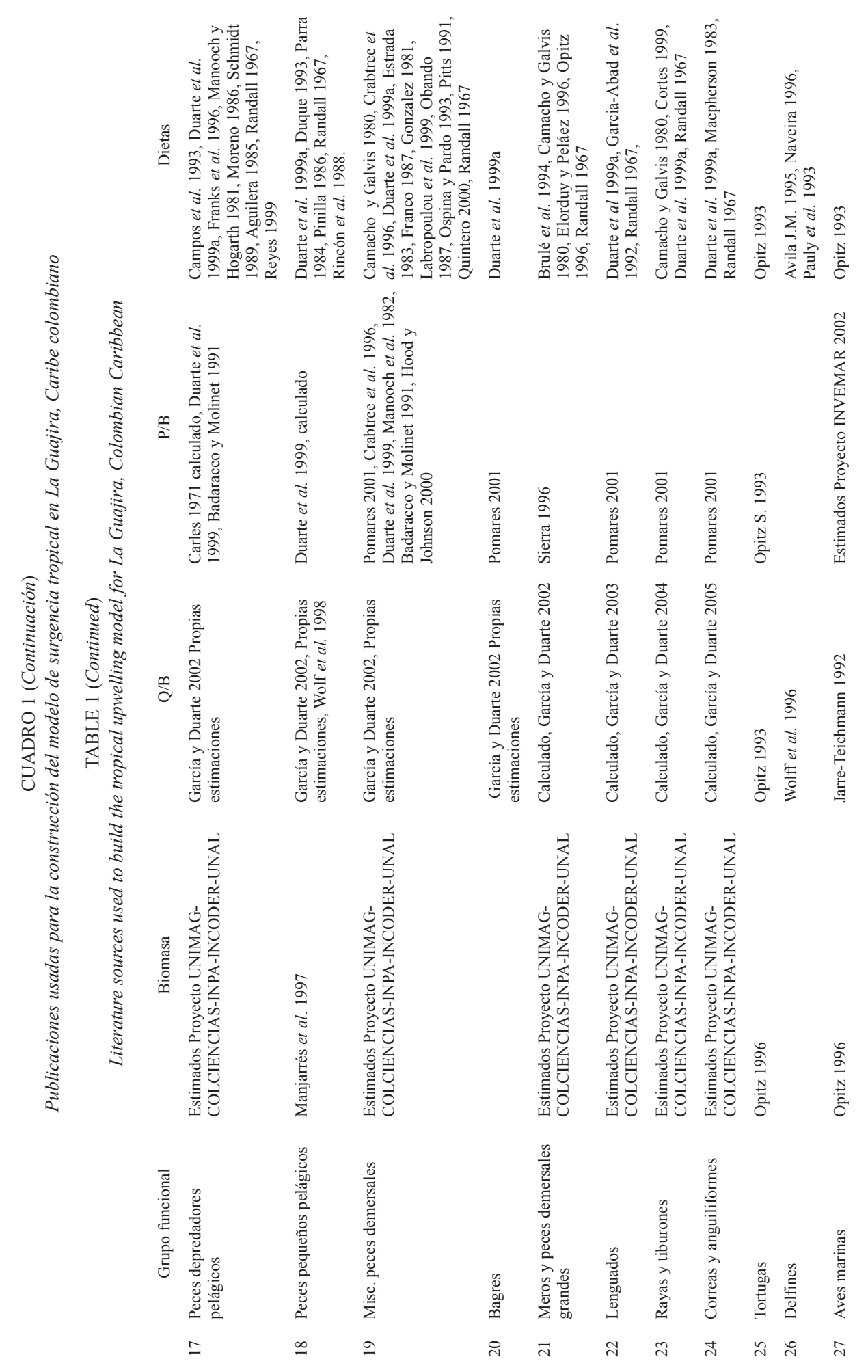




\section{CUADRO 2}

Grupos funcionales asignados para el modelo ECOPATH de surgencia tropical en La Guajira, Caribe colombiano, se detallan las especies o familias.

TABLE 2

Functional groups of species and families in tropical upwelling ECOPATH model. La Guajira, Colombian Caribbean

\section{Grupos} funcionales
Especies o familias asignadas
Fuente Bibliográfica
1 Fitoplancton

2 Macroalgas
3 Pastos marinos

4 Zooplancton

5 Poliquetos
Fitoplancton

Osmundaria obtusiloba, Amphiroa rígida, Antithamnionella breviramosa, Botryocladia occidentalis, Bryopsis pennata, Caulerpa cupressoiodes, Caulerpa mexicana Sonácrex, Caulerpa racemosa var. Peltata, Ceramium nitens, Codium decorticatum, Codium decorticatum, Caulerpa mexicanaSonácrex, Corallinaceae sp., Crouania attenuata, Dasya mollis, Dictyopterís delicatula, Dictyosa cf. hartayresiana, Dictyota cervicomis, Dictyota pulchella, Gracilaria cylindrica, Gracilaria dominguensis, Gracilaria mamillaria, Gracilaria sp. 2, Gracilaria sp. 3, Gracilaria sp., Haloplegma duperreyi, Heterosiphonía gibbesii, Hydrolithon boergesenii, Hypoglossum simutam, Jania adhaerem, Laurencia sp., Lejolisia cf. mediterránea, Polyüphonia denudata, Spyridia filamentosa, Wrangellia penicillata, Caulerpa prolifera, Caulerpa sertularioides, Caulerpella ambigua, Avramvillea sp., Halimeda gracilis, Halimeda incrassata, Udotea caribaea, Udotea conglutinata, Udotea flabellum.

Thallasia testudinium, Syringodium filiforme, Halodule wrighti

Amphipoda, Cladocera, Copepoda, Crustacea (Larvas), Chaetognatha, Echinoidea, Euphasiacea, Foraminifera, Hydromedusae, Mollusca, Ostracoda, Polychaeta, Sergestida, Thaliacea.

Acoetidae, Aphrodita acuminata, Benthoscolex cubanus, Capitellidae, Ceratonereis irritabilis, Chloeia viridis, Eunice biannulata, Eunice hawaiensis, Eunice multicylindri, Eunice panamena, Harmothoe sp.

Hyalinoecia artifex, Megelonidae, Neanthes sp., Pareurythoe sp. Spionidae, Sternaspis scutata, Travisia $\mathrm{sp.}$

6 Langostas Panulirus argus, Panulirus laevicauda, Panulirus guttatus.

7 Cangrejos y otros Callinectes crustáceos

8 Camarones

9 Pulpos y calamares

10 Asteroideas y ofiuros
Xiphonopeneus kroyeri, Penaeus subtilis, Penaeus brasiliensis, Penaeus schmitti, Pennaeus notialis, Rimapenaeus similis.

Teuthoidea, Octopus vulgaris, Octopus macrurus

Amphilimna olivacea, Amphiodia atra, Amphipholizona delicata, Anthenoides piercei, Astrocnida isidis, Astropecten alligator, Astropecten antillensis, Luidia heterozona barimae, Ophimyces frutectosus, Ophiolepis elegans, Ophiomixa tumida, Ophiomusium acuferum, Ophionereis dolabriformis, Ophiopaepale goesiana, Ophiophragmus riisei, Ophiopsila hartmeyeri, Ophiothrix angulata, Ophiura acervata, Oreaster reticulatus, Tosia parva.
Díaz-Pulido 2002

Diaz et al. 2003

Bernal 2000

Guzman-Alvis y Solano 1997, Navas et al. 2002

Correa y Manjarrés 2002, Viaña et al. 2004

Correa y Manjarrés 2004, Viaña et al. 2004

Correa y Manjarrés 2004, Viaña et al. 2004

Bedoya et al. 2000

Navas et al. 2002 
CUADRO 2 (Continuación)

Grupos funcionales asignados para el modelo ECOPATH de surgencia tropical en La Guajira, Caribe colombiano, se detallan las especies o familias.

TABLE 2 (Continued)

Functional groups of species and families in tropical upwelling ECOPATH model. La Guajira, Colombian Caribbean

Grupos funcionales

11 Miscelánea de invertebrados herbívoros

12 Invertebrados carnívoros

13 Pámpano, bonito y jurel

14 Pargos

15 Corvinas

16 Peces depredadores pelágicos

17 Peces pequeños pelágicos
Especies o familias asignadas

Bivalvia, Acesta colombiana, Adrana tellinoides, Anadara sp., Aplysinidae, Arca zebra, Argopecten, Barbatia candida, Corbula sp., Corbula chittyana, Chlamys munda, Entodesma beana, Laevicardium pictum, Laevicardium sybariticum, Lythechinus variegatus, Macoma tenta, Myxillidae, Nemocardium tinctum, Niphatidae, Nuculana cestrota, Pecten chazaliei, Petrosiidae, Pitar arestus, Spirastrellidae, Spirastrellidae, Tehtyidae, Tellina probrina-consobrina, Thorectidae

Equinodermos (Ancilla glabrata, Brissopsis elongata, Clypeaster euclastus, Cochlespira radiata, Cosmioconcha nitens, Eucidaris tribuloides) Gastropodos (Latirus mcgintyi, Murex sp., Nassarius hotessieri, Oliva scripta, Olivella lactea, Persicula pulcherrima, Polystira albida, Sinum maculatum, Stylocidaris affinis, Vermicularia spirata).

Trachinotus carolinus, Euthynnus alletteratus Caranx hippos.

Conodon nobilis, Haemulon bonariense, Haemulon plumieri, Lachnolaimus maximus, Lutjanus analis, Lutjanus bucanela, L. crysurus, L. griseus, L. jocu, L. mahogoni, L. purpureus, L. synagris, L. vivanus, Orthopristis ruber, Pristipomoides aquilonaris, Rhomboplites aurorubens.

Cynoscion acoupa, C. jamaicensis, C. leiarchus, Menticirhus americanus, M. litoralis, Micropogonias furnieri, Umbrina coroides.

Ablennes hians, Acanthocibium solandri, Albula vulpes, Alectis ciliaris, Auxis thazard, Caranx bartholomaei, Caranx crysos, C. latus, C. ruber, Coryphaena hippurus, Chaetodipterus faber, Decapterus macarellus, Elagatis bipinnulata, Elops saurus, Hemicaranx amblyrhynchus, Istiophorus albicans, Lobote surinamensis, Pomatomus saltatrix, Rachycentron canadum, Scomberomorus brasiliensis, Scomberomorus cavalla, Scomberomorus regalis, Selar crumenophthalmus, Selene setapinnis, Selene vomer, Sphyraena barracuda, Sphyraena guachancho, Tarpon atlanticus, Trachinotus falcatus, Trachinotus goodei, Trachurus lathami, Tylosurus spp., Uraspis secunda.

Anchoa sp., Chloroscombrus chrysurus, Decapterus sp., Engraulis eurystole, Harengula jaguana, Lycengraulis grossidens, Oligoplites palometa, Oligoplites saliens, Oligoplites saurus, Opistonema oglinum, Sardinella aurita, Scomber japonicus.
Fuente Bibliográfica

Navas et al. 2002,

Montoya-Maya 2002.

Navas et al. 2002

Correa y Manjarrés 2004 Viaña et al. 2004

Correa y Manjarrés 2004, Viaña et al. 2004

Correa y Manjarrés 2004 Viaña et al. 2004

Correa y Manjarrés 2004, Viaña et al. 2004

Correa y Manjarrés 2004, Viaña et al. 2004 
CUADRO 2 (Continuación)

Grupos funcionales asignados para el modelo ECOPATH de surgencia tropical en La Guajira, Caribe colombiano, se detallan las especies o familias.

TABLE 2 (Continued)

Functional groups of species and families in tropical upwelling ECOPATH model. La Guajira, Colombian Caribbean

Grupos funcionales
Especies o familias asignadas

18 Peces demersales
Acanthostracion bicaudalis, A. polygonius, A quadricornis, A. trigonus, Acanthurus chirurgus, Aluterus heudelotii, A. monoceros, A. schoepfii, Anisotremus surinamensis, A. virginicus, Antennarius striatus, Apogon affinis, A. pseudomaculatus, A. quadrisquamatus, Archosargus rhomboidalis, Bairdiella sanctaeluciae, Balistes carolinensis, B. vetula, Bellator ribeiroi, Calamus penna, $C$. pennatula, Canthigaster rostrata, Colomesus sp., Ctenosciaena gracilicirrhus, Chaetodon ocellatus, Ch.sedentarius, Ch. striatus, Chilomycterus antillarum, Ch. reticulatus, Chirocentrodon bleekerianus, Diapterus auratus, D. olisthostomus, D. rhombeus, Diodon holocanthus, Diodon sp., Diplectrum bivittatum, D. formosum, D. radiale, Echeneis naucrates, E. neucratoides, Equetus acuminatus, E. lanceolatus, Eucinostomus argenteus, E. gula, E. harengulus, E. melanopterus, Euguerres plumieri, Gerres cinereus, Haemulon aurolineatum, H. boschmae, H. carbonarium, $H$. chrysargyreum, $H$. flavolineatum, $H$. parrai, H. melanurum, H. steindachneri, H. striatum, Halieutichthys aculeatus, Holocentrus ascensionis, H. rufus, Isopisthus parvipinnis, Lagocephalus laevigatus, Larimus breviceps, Macrodon ancylodon, Monacanthus setifer, Mugil curema, M. incilis, M. liza, Mulloidichthys martinicus, Mullus auratus, Myripristis jacobus, Nebris microps, Nicholsina usta, Ogcocephalus parvus, Ogcocephalus sp., Orthopristis ruber, Pagrus pagrus, Paralonchurus brasiliensis, Polydactylus virginicus, Pomacanthus arcuatus, P. paru, Pomadasys corvinaeformis, Porichthys plectrodon, Priacanthus arenatus, Prionotus ophryas, P. punctatus, Pristigenys alta, Pseudupeneus maculatus, Saurida brasiliensis, S. normani, Scorpaena brasiliensis, S. calcarata, S. isthmensis, $S$. plumieri, Serranus phoebe, Sparisoma sp., Sphoeroides dorsalis, Sphoeroides spengleri, Stellifer microps, Stellifer rastrifer, Synodus foetens, Synodus intermedius, S. poeyi, Thalassophryne maculosa, Trachinocephalus myops, Umbrina broussonnettii, Upeneus parvus.

19 Bagres

20 Meros y peces demersales grandes

21 Lenguados
Arius grandicassis, Arius proops, Bagre marinus, Cathorops spixi.

Caulolatilus cyanops, C. guppyi, Centropomus undecimalis, Ephinephelus adscencionis, E. flavolimbatus, E. guttatus, E. itajara, E. morio, E. niveatus, Fistularia petimba, F. tabacaria, Halichoeres caudalis, Kyphosus spp, Mycteroperca bonasi, Seriola dumerili, S. rivoliana, S. zonata, Xirichtys novacula

Acylopsetta kumperae, Bothus ocellatus, Bothus robinsi, Cyclopsetta chittendeni, Etropus crossotus, Gastropsetta frontalis, Gymnachirus nudus, Paralichthys tropicus, Syacium micrurum, Syacium papillosum, Symphurus tessellatus, Trinectes paulistanus.
Fuente Bibliográfica

Correa y Manjarrés 2004, Viaña et al. 2004

(1)

Correa y Manjarrés 2004, Viaña et al. 2004

Correa y Manjarrés 2004, Viaña et al. 2004

Correa y Manjarrés 2004, Viaña et al. 2004 
CUADRO 2 (Continuación)

Grupos funcionales asignados para el modelo ECOPATH de surgencia tropical en La Guajira, Caribe colombiano, se detallan las especies o familias.

TABLE 2 (Continued)

Functional groups of species and families in tropical upwelling ECOPATH model. La Guajira, Colombian Caribbean

\begin{abstract}
Grupos
funcionales

Especies o familias asignadas

Aetobatus narinari, Carcharhinus acronotus, C. signatus, Carcharinus spp, Dasyatis americana, D. guttata, Diplobatis pictus, Ginglymostoma cirratum, Mustelus canis, M. higmani, M. norrisi, Myliobatis sp, M. freminvillei, Diplobatis pictus, Narcine brasiliensis, Rhinobatos percellens, Rhinoptera bonosus, Rhizoprionodon porosus, Rhizoprionodon spp, Squatina dumeril, Urobatis jamaicensis.
\end{abstract}

2 Rayas y tiburones
23 Anguiliformes

24 Tortugas

25 Delfines

26 Aves

27 Detritus
Brotula barbata, Cynoponticus savanna, Echiophis punctifer, Enchelycore nigricans, Gymnothorax conspersus, G. ocellatus, Hoplunnis schmidti, Lepophidium brevibarbe, L. pheromystax, $L$. profundorum, Lycodontis moringa, Ophichthus ophis, O. parilus, Ophidion holbrooki, Paraconger caudilimbatus, Rynchonger flavus, Trichiurus lepturus.

Caretta caretta, Dermochelys coriacea, Eretmochelys imbricata.

Delfines

Pelícanos

Detritus
Fuente Bibliográfica

Correa y Manjarrés 2004, Viaña et al. 2004

Correa y Manjarrés 2004, Viaña et al. 2004

Correa y Manjarrés 2004, Viaña et al. 2004

Vergara 1990 niveles tróficos discretos sensu como lo sugiere Ulanowicz (1986), y de esta forma se puede estimar la distribución de la biomasa entre los niveles tróficos y las eficiencias en las transferencias. Algunos índices de los flujos, como son las transferencias totales ( $\mathrm{T}$ ), y el índice de ciclaje de Finn (FCI) fueron utilizados para representar el grado de madurez del ecosistema (Finn 1976). También se incluyó en los resultados la ascendencia (A) que representa el grado de desarrollo del sistema en términos de flujos totales $(\mathrm{T})$ y contenido de información (I) (Ulanowicz 1986).

\section{RESULTADOS}

Los parámetros de entrada y los estimados después de balancear el modelo se resumen en los Cuadros 3 y 5 . Los principales flujos de materia a la respiración (R) se dan en el zooplancton, miscelánea de invertebrados herbívoros, poliquetos y peces pelágicos pequeños, los cuales presentan a su vez en el ecosistema estimaciones altas en la biomasa (Cuadro 5). Se debe resaltar que en la relación respiración/ asimilación (R/A) el zooplancton presenta los valores más bajos de todos los grupos funcionales debido a alta tasa de renovación de este grupo. En los demás grupos los valores encontrados son más elevados y guardan relación con la fisiología de los grupos, de esta manera los homeotermos, que son las aves y delfines, presentan los valores más altos de R/A.

Según Odum y Heald (1975) la cadena trófica es un conjunto de niveles tróficos (categorizados por el tipo de alimento que los organismos consumen), donde los productores primarios y el detritus se encuentran ubicados en el nivel trófico I. Los poliquetos, miscelánea de invertebrados herbívoros, zooplancton, cangrejos y crustáceos, camarones y tortugas 
CUADRO 3

Datos básicos de entrada al modelo de sistema de surgencia tropical de La Guajira en el Caribe colombiano

TABLE 3

Basic input parameters of the mass balance mode, tropical upwelling system, Colombian Caribbean

\begin{tabular}{|c|c|c|c|c|c|c|c|c|c|}
\hline Grupo Funcional & $\begin{array}{l}\text { Nivel } \\
\text { Trófico }\end{array}$ & $\begin{array}{l}\text { Área de } \\
\text { hábitat }\end{array}$ & $\begin{array}{c}\text { Biomasa en } \\
\text { área de hábitat } \\
\qquad\left(\mathrm{t} / \mathrm{km}^{2}\right)\end{array}$ & $\begin{array}{l}\text { Biomasa } \\
\left(\mathrm{t} / \mathrm{km}^{2}\right)\end{array}$ & $\begin{array}{l}\text { Prod./ } \\
\text { biom. } \\
\text { (/year) }\end{array}$ & $\begin{array}{l}\text { Cons./ biom. } \\
\text { (/year) }\end{array}$ & $\begin{array}{l}\text { Eficiencia } \\
\text { ecotrófica }\end{array}$ & $\begin{array}{c}\text { Producción / } \\
\text { consumo }\end{array}$ & $\begin{array}{c}\text { Capturas } \\
\left(\mathrm{t} / \mathrm{km}^{2}\right)\end{array}$ \\
\hline Fitoplancton & 1 & 1 & (12.328) & $(12.328)$ & 70 & - & 0.8 & - & \\
\hline Macroalgas & 1 & 0.009 & 122.5 & 1.102 & 17 & - & $(0.806)$ & - & \\
\hline Pastos marinos & 1 & 0.034 & 842.85 & 28.657 & 12.8 & - & $(0.121)$ & - & \\
\hline Zooplancton & 2.33 & 1 & 9.68 & 9.68 & 40 & (88.404) & 0.7 & $(0.452)$ & \\
\hline Poliquetos & 2.03 & 1 & $(0.751)$ & $(0.751)$ & 15 & 80 & 0.919 & $(0.188)$ & \\
\hline Langostas & 3.4 & 1 & $(1.404)$ & (1.404) & 1.02 & 7 & 0.96 & $(0.146)$ & 0.05 \\
\hline $\begin{array}{l}\text { Cangrejos y otros } \\
\text { crustáceos }\end{array}$ & 2.45 & 1 & 1.58 & 1.58 & 3.5 & 16 & $(0.881)$ & $(0.219)$ & 0.03 \\
\hline Camarones & 2.42 & 1 & 0.16 & 0.16 & 5.1 & 25 & $(0.988)$ & $(0.204)$ & 0.16 \\
\hline Pulpos y calamares & 3.66 & 1 & 0.11 & 0.11 & 2.3 & 8 & $(0.957)$ & $(0.288)$ & 0.03 \\
\hline $\begin{array}{l}\text { Asteroideas y } \\
\text { ofiuros }\end{array}$ & 3.17 & 1 & 0.75 & 0.75 & 0.9 & 3 & $(0.961)$ & $(0.3)$ & 0.09 \\
\hline $\begin{array}{l}\text { Invertebrados } \\
\text { herbívoros }\end{array}$ & 2.29 & 1 & 4.5 & 4.5 & 3.8 & 29.1 & $(0.914)$ & $(0.131)$ & 0.18 \\
\hline $\begin{array}{l}\text { Invertebrados } \\
\text { carnívoros }\end{array}$ & 2.77 & 1 & 2.5 & 2.5 & 2.3 & 8 & $(0.992)$ & $(0.288)$ & 0.06 \\
\hline $\begin{array}{l}\text { Pámpano, Bonito } \\
\text { y Jurel }\end{array}$ & 3.67 & 1 & 0.13 & 0.13 & $(2.412)$ & 10.3 & 0.95 & $(0.234)$ & 0.13 \\
\hline Pargos & 3.7 & 1 & 0.43 & 0.43 & 0.56 & 4.5 & $(0.561)$ & $(0.124)$ & 0.09 \\
\hline Corvinas & 3.51 & 1 & 0.03 & 0.03 & (1.83) & 9.15 & $(0.622)$ & 0.2 & 0.02 \\
\hline $\begin{array}{l}\text { Peces depre-dadores } \\
\text { pelágicos }\end{array}$ & 3.71 & 1 & 0.17 & 0.17 & 1.95 & 10.3 & $(0.774)$ & $(0.189)$ & 0.06 \\
\hline $\begin{array}{l}\text { Peces pelágicos } \\
\text { pequeños }\end{array}$ & 2.74 & 1 & 2.73 & 2.73 & 4 & 14.6 & $(0.311)$ & $(0.274)$ & 0.01 \\
\hline Peces demersales & 3.19 & 1 & 0.88 & 0.88 & 3.17 & 10 & $(0.849)$ & $(0.317)$ & 0.4 \\
\hline Bagres & 3.22 & 1 & $(0.013)$ & $(0.013)$ & 1.3 & 7 & 0.95 & $(0.186)$ & 0.01 \\
\hline $\begin{array}{l}\text { Meros y peces } \\
\text { demersales grandes }\end{array}$ & 3.79 & 1 & 0.092 & 0.092 & $(1.32)$ & 6.6 & $(0.616)$ & 0.2 & 0.02 \\
\hline Lenguados & 3.38 & 1 & 0.14 & 0.14 & 0.83 & 7.5 & $(0.931)$ & $(0.111)$ & 0.04 \\
\hline Rayas y tiburones & 3.68 & 1 & 0.09 & 0.09 & 0.62 & 5.3 & $(0.858)$ & $(0.117)$ & 0.04 \\
\hline $\begin{array}{l}\text { Correas y } \\
\text { anguiliformes }\end{array}$ & 3.84 & 1 & 0.11 & 0.11 & $(0.749)$ & 4.8 & 0.94 & $(0.156)$ & 0.01 \\
\hline Tortugas & 2.67 & 1 & 0.04 & 0.04 & 0.3 & 3.5 & $(0.839)$ & $(0.086)$ & 0 \\
\hline Delfines & 4 & 1 & 0.021 & 0.021 & 0.05 & 28 & 0 & $(0.002)$ & \\
\hline Aves & 4.16 & 1 & 0.006 & 0.006 & 0.7 & 39.33 & 0 & $(0.018)$ & \\
\hline Detritus & 1 & 1 & - & - & - & - & 0.117 & - & \\
\hline
\end{tabular}

Los valores en paréntesis fueron estimados por Ecopath. Values in brackets were estimated with the Ecopath model. 
se encuentran situados en fracciones del nivel trófico II (Cuadro 4). La dieta de estos grupos consiste básicamente de productores primarios y/o detritus. Los demás grupos funcionales se encuentran en fracciones del nivel trófico III, y las aves y delfines conforman fracciones del nivel trófico superior IV. Estos depredadores superiores se alimentan principalmente de los pequeños pelágicos y de los peces demersales (Fig. 2).

Los grupos funcionales que contribuyen significativamente al detritus son zooplancton, pastos marinos y fitoplancton (Cuadro 5), el $17 \%$ de la materia del detritus que se produce en el ecosistema es transferido a los siguientes tres niveles tróficos lo demás es sepultado en el sedimento o exportado. El resumen estadístico (Cuadro 6) muestra el tamaño del ecosistema de surgencia de La Guajira, y puede ser usado para compararlo con otros ecosistemas similares. Además, el Cuadro incluye un número de índices acerca de los flujos de la red. Las transferencias totales del sistema están por encima de $3000 \mathrm{t} / \mathrm{km}^{2} / \mathrm{año}$, el $35.61 \%$ es destinado al consumo, el $14.90 \%$ a la respiración, el $23.22 \%$ es exportado fuera del sistema y el $26.26 \%$ es llevado al detritus (Cuadro 6). En términos generales el ecosistema presenta características propias de sistemas de surgencia, por ejemplo, el volumen de biomasa que se produce en pequeños pelágicos y productores primarios, el cual no puede ser incorporado directamente en su totalidad a la red alimentaria y como consecuencia este material cae al sedimento o es exportado fuera del sistema. Siguiendo los análisis de Ecopath, se puede observar en cada nivel trófico los flujos relativos y el rendimiento en la transferencia de energía (Fig. 3). Un acercamiento a la distribución de los flujos, señala que el Nivel Trófico II (herbívoros), presenta el mayor porcentaje en las transferencias (Fig. 3).

El detritus tiene un impacto positivo sobre gran parte de los grupos funcionales especialmente en los invertebrados. La producción de pequeños pelágicos tiene un impacto positivo sobre tortugas, delfines, correas y anguiliformes. Los otros grupos no muestran un impacto significativo positivo o negativo sobre alguno de los grupos, como se ve en las pequeñas barras o ausencia de éstas sobre las correspondientes líneas. Las flotas pesqueras que operan en el área están causando detrimento de rayas y tiburones. La flota artesanal ejerce un impacto negativo sobre más grupos que las otras flotas mientras que la flota industrial afectan negativamente a las corvinas. (Fig. 4). Las diferentes flotas pesqueras que trabajan en La Guajira equivalen a un depredador más dentro del ecosistema, por consiguiente presentan un nivel trófico medio de 3.11 (Cuadro 6), lo que significa que extraen recursos entre los niveles II y III. La mortalidad por pesca de los pargos, corvinas, rayas y tiburones presenta valores superiores a la mortalidad por depredación ( 0.2 a 0.1 en pargos, 0.9 a 0.5 en corvinas y 0.48 a 0.05 en rayas y tiburones, respectivamente). Este resultado es útil para establecer predicciones acerca de los cambios en las abundancias de estos grupos, como consecuencia de la fuerte presión pesquera de la que son objeto.

Los depredadores contribuyen en un $13.85 \%$ de los ciclos en los flujos de biomasa cuando el detritus fue excluido. El valor de índice de ciclos de Finn fue $7.47 \%$ (con respecto al total de las transferencias totales) y el promedio de la longitud de las vías de Finn 2.62; el índice de conectancía fue de 0.30 y el índice de omnivoría del sistema 0.31 . Los valores de ascendencia, estabilidad, capacidad e información se muestran en el Cuadro 6. Estos valores proveen información acerca de la capacidad de reserva y permanencia ante eventos inesperados de perturbación (Ulanowicz 1986). Los valores encontrados en la estabilidad sugieren que el ecosistema tiene una alta resiliencia, y ya que la capacidad de desarrollo está por encima del límite encontrado en la medida de ascendencia, aparentemente La Guajira es un sistema con un alto potencial de crecimiento trófico.

\section{DISCUSIÓN}

El modelo del ecosistema de La Guajira presenta variabilidad en la exactitud y precisión 


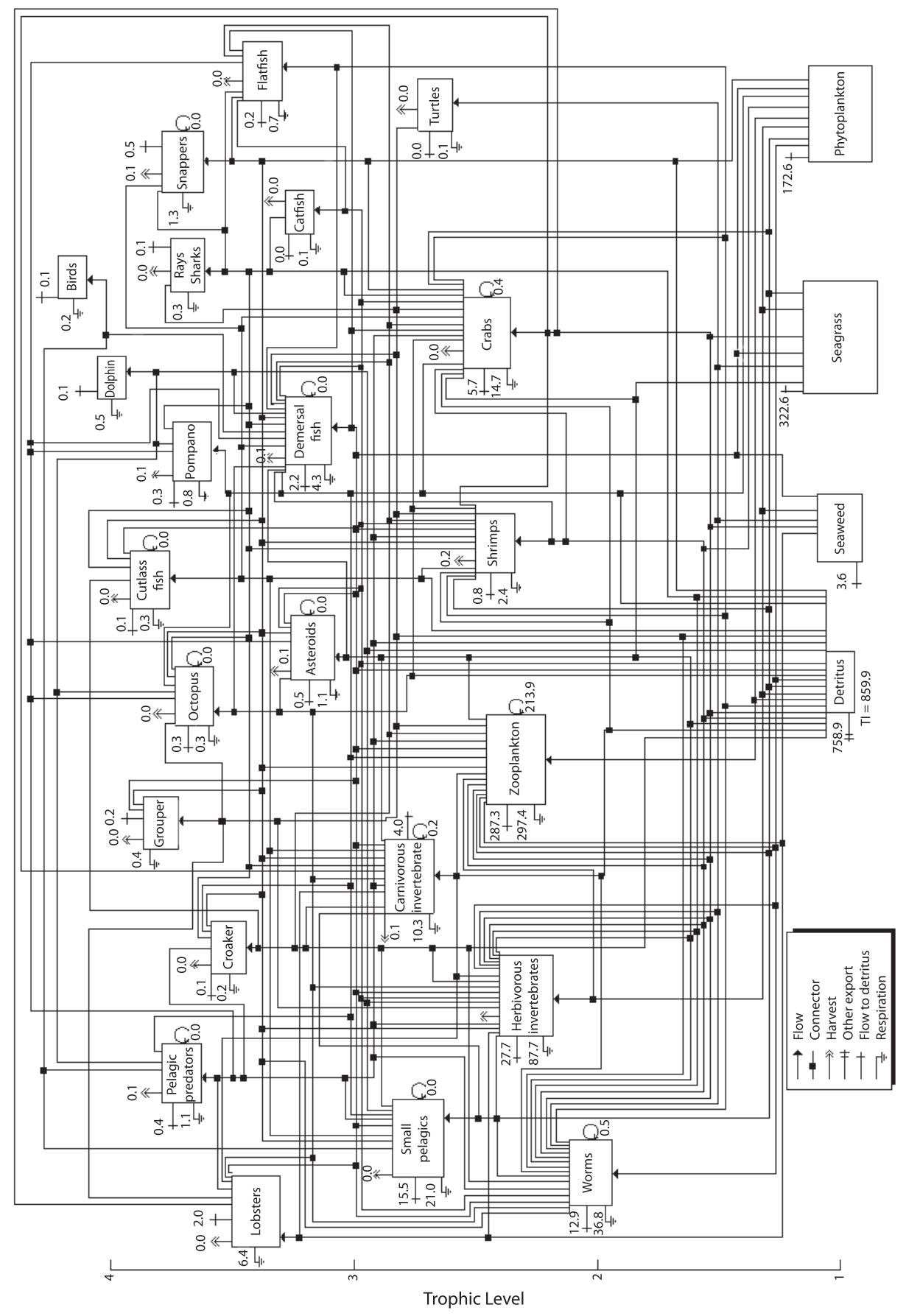

Fig. 2 Diagrama de los flujos de materia y energía en el sistema de surgencia tropical de La Guajira, Caribe colombiano. Los componentes del sistema están estructurados a lo largo del eje vertical de acuerdo a su nivel trófico definido como 1 para productores primarios y detritus

Fig. 2. Flow diagram of the upwelling tropical system off La Guajira, Caribbean Colombian. The components of the system are structured along the vertical axis according to their trophic level defined as 1 for primary producers and detritus. 


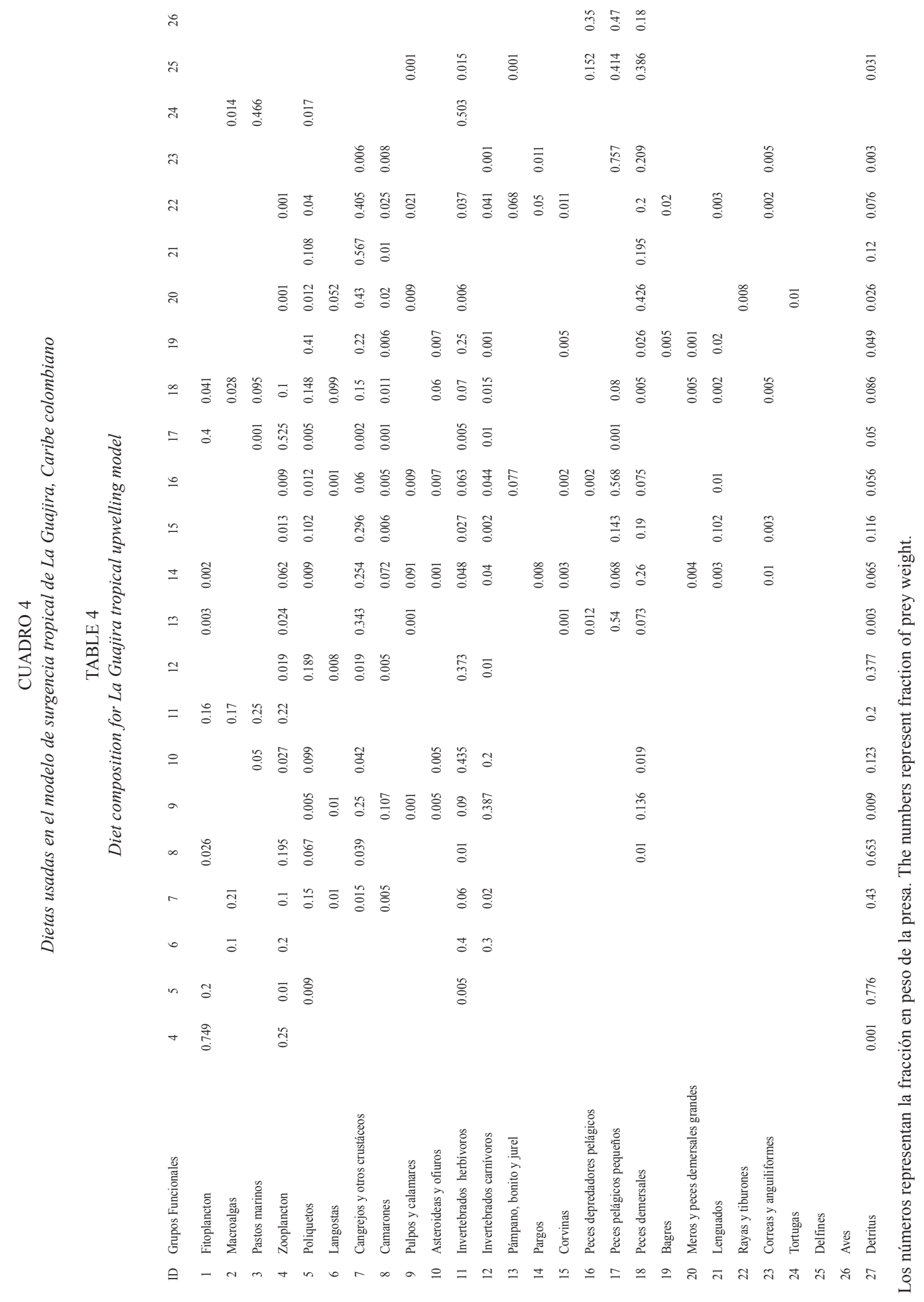


CUADRO 5

Principales resultados de los parámetros estimados en la construcción del modelo de surgencia tropical, por grupo funcional

TABLE 5

Main results of estimated parameters in the construction of the tropical upwelling model, by functional group

Grupo Funcional

Fitoplancton

Macroalgas

Pastos marinos

Zooplancton

Poliquetos

Langostas

Cangrejos y otros crustáceos

Camarones

Pulpos y calamares

Asteroideas y ofiuros

Misc. invert. herbívoros

Misc. invert. carnívoros

Pámpano, bonito y jurel

Pargos

Corvinas

Peces depredadores pelágicos

Peces pelágicos pequeños

Misc. peces demersales

Bagres

Meros y peces demersales grandes

Lenguados

Rayas y tiburones

Correas y anguiliformes

Tortugas

Delfines

Aves

Detritus

$\begin{array}{ccc}\text { Nivel } & \text { Respiración } & \text { Asimilación } \\ \text { trófico } & \left(\mathrm{t} / \mathrm{km}^{2} / \mathrm{año}\right) & \left(\mathrm{t} / \mathrm{km}^{2} / \mathrm{año}\right)\end{array}$

1

1

1

2.33

2.03

3.4

2.45

2.42

3.66

3.17

2.29

2.77

3.67

3.7

3.51

3.71

2.74

3.19

3.22

3.79

3.38

3.68

3.84

2.67

4

4.16

1

0
0
0

297.402

36.809

6.429

14.694

2.384

0.319

1.125

87.66

10.25

0.758

1.307

0.165

1.069

20.966

4.25

0.056

0.364

0.724

0.326

0.34

0.1

0.469

0.185

0

684.602
48.078

7.86

20.224

3.2

0.572

1.8

104.76

16

1.071

1.548

0.22

1.401

31.886

7.04

0.073

0.486

0.84

0.382

0.422

0.112

0.47

0.189
$\begin{array}{ccc}\mathrm{R} / \mathrm{A} & \begin{array}{c}\text { Flujos a detritus } \\ \left(\mathrm{t} / \mathrm{km}^{2} / \mathrm{año}\right)\end{array} & \begin{array}{c}\text { Eficiencia } \\ \text { neta }\end{array}\end{array}$

172.587

3.644

322.587

287.311

0.566

0.434

12.932

0.234

0.818

0.182

0.727

2.022

0.273

0.745

5.717

0.255

0.558

0.81

0.442

0.625

0.319

0.375

0.837

0.476

0.163

0.641

27.658

0.359

0.707

4.045

0.293

0.844

0.283

0.156

0.75

0.493

0.25

0.763

0.076

0.237

0.658

0.425

0.342

0.604

2.181

0.396

0.768

0.019

0.232

0.75

0.168

0.25

0.862

0.218

0.138

0.854

0.103

0.146

0.805

0.111

0.195

0.893

0.03

0.107

0.998

0.119

0.002

0.978

0.051

0.022 de las series de datos usados de entrada debido a limitaciones en la información para el área. Este déficit en la exactitud de los datos de entrada genera innumerables posibles combinaciones de los parámetros que describen el ecosistema, pero los resultados del balance del modelo que aquí se encontraron resultan razonables para explicar la dinámica del ecosistema. Al evaluar la calidad de los resultados se encontró que la eficiencia bruta (GE) para peces e invertebrados están dentro de los ámbitos presentados por Mann (1982) y Caddy y 
CUADRO 6

Resumen estadístico del sistema de surgencia tropical de La Guajira, Caribe colombiano

TABLE 6

Statistical summary for the tropical upwelling system, La Guajira, Colombian Caribbean

\section{Parámetro}

Suma de todos los consumos

Suma de todas las exportaciones

Suma de total de los flujos a respiración

Suma de todos los flujos al detritus

Transferencias totales del sistema

Suma de toda la producción

Promedio del nivel trófico de captura

Rendimiento bruto de la pesquería(captura/ppn)

Producción primaria neta (ppn)

Producción primaria total/ respiración total

Producción neta del sistema

Producción primaria total/biomasa total

Biomasa total/transferencias totales

Biomasa total (sin detritus)

Capturas totales

Índice de conexión

Índice de los ciclos de Finn

Promedio en la longitud de viás de Finn

Índice de omnivoría del sistema

Ascendencia

Estabilidad

Capacidad

Información

Rendimiento de transferencias (RT) (\%) por nivel trófico

\begin{tabular}{|c|c|}
\hline Valor & Unidades \\
\hline 1166.341 & $\mathrm{t} / \mathrm{km}^{2} / \mathrm{año}$ \\
\hline 760.527 & $\mathrm{t} / \mathrm{km}^{2} / \mathrm{año}$ \\
\hline 487.984 & $\mathrm{t} / \mathrm{km}^{2} /$ año \\
\hline 860.257 & $\mathrm{t} / \mathrm{km}^{2} / \mathrm{año}$ \\
\hline 3275 & $\mathrm{t} / \mathrm{km}^{2} /$ año \\
\hline 1693 & $\mathrm{t} / \mathrm{km}^{2} / \mathrm{año}$ \\
\hline 3.11 & \\
\hline 0.0011 & \\
\hline 1248.511 & $\mathrm{t} / \mathrm{km}^{2} /$ año \\
\hline 2.559 & \\
\hline 760.527 & $\mathrm{t} / \mathrm{km}^{2} / \mathrm{año}$ \\
\hline 18.279 & \\
\hline 0.021 & \\
\hline 68.302 & $\mathrm{t} / \mathrm{km}^{2}$ \\
\hline 1.43 & $\mathrm{t} / \mathrm{km}^{2} / \mathrm{año}$ \\
\hline 0.305 & \\
\hline 7.47 & $\%$ del total de las transferencias \\
\hline 2.62 & \\
\hline 0.314 & \\
\hline 4095.3 & \\
\hline 8078.5 & \\
\hline 12173.8 & \\
\hline
\end{tabular}

1.251

Productores

II III IV $\quad$ V VI $\quad$ VII

Detritus

9.4

15.7

12.8

$\begin{array}{lll}18.3 & 15.5 & 14.9\end{array}$

12.1

Todos los flujos

10.1

19.5

$\begin{array}{lll}15.9 & 15.8 & 11.9\end{array}$

12.5

Proporción de todos los flujos originados desde el detritus

14

$17.7 \quad 15.6 \quad 14.3$

0.32

$13 \%$

Eficiencia en las transferencias desde los Productores Primarios

$17 \%$

Eficiencia en las transferencias desde el detritus 


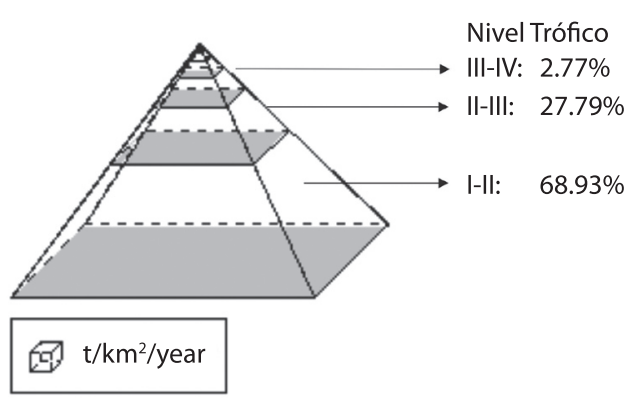

Fig. 3. Pirámide trófica de los flujos en el ecosistema de La Guajira. El volumen de cada nivel trófico es proporcional a las transferencias totales en cada nivel. El ángulo de la pirámide es inversamente proporcional al promedio en la eficiencia de transferencias del sistema. El compartimiento de la base representa el nivel II.

Fig. 3. La Guajira tropical upwelling system. Modified Lindeman pyramid of flows; the volume of each discrete trophic level is proportional to the throughput (total flow) at that level; the bottom compartment represents trophic level II.

Sharp (1988); para el zooplancton, como presenta un metabolismo más alto, el valor de GE es superior a 0.3 debido a la activa herbivoría. Existe evidencia en organismos planctónicos pequeños y en especies tropicales que el valor de GE es más alto que el de organismos grandes (Tranter 1976). La tasa de respiración sobre asimilación (R/A) en todos los grupos está dentro de los valores registrados en la literatura (Humphreys 1979, Valiela 1995).

Las biomasas calculadas por el modelo para los grupos de invertebrados son mayores que las estimadas en los muestreos a bordo de los barcos arrastreros (Viaña et al. 2004) y que las abundancias encontradas por Navas et al. (2002). Estos grupos usualmente resultan subestimados en las capturas con redes de pesca experimental o comercial; otros autores han encontrado que el margen de error cometido por el uso de redes de arrastre puede ser de un $50 \%$ del valor obtenido (Badaracco y Molinet 1991, Wolff et al. 1998). En los invertebrados herbívoros e invertebrados carnívoros la subestimación fue la más alta, los valores de biomasa estimados inicialmente resultaron inferiores a la biomasa que es consumida por los depredadores de estos grupos según el modelo. Otro aspecto importante es el hecho de que las estaciones muestreadas en las campañas científicas de macrofauna (Navas et al. 2002) no incluyeron el área de la pradera de Thalassia, lo que introduce un sesgo en las estimaciones de biomasa de los grupos que hacen parte de esta comunidad. Se ha documentado que las praderas de pastos juegan un rol importante como hábitat de gran número de organismos infaunales, especialmente bivalvos detritívoros (Díaz y Puyana 1994), y de los cuales no existe antecedentes acerca de los valores de biomasa en el área de estudio. Las estimaciones iniciales de biomasa en estos grupos que fueron modificadas en el modelo, resultaron razonables, según lo sugerido por Mann (1982). No obstante, es necesario validar estos resultados con investigaciones tendientes a cuantificar las densidades de los invertebrados en el área de estudio.

Los resultados indican que el ecosistema tiene un notable suministro de materia y energía a través de los pastos marinos, el fitoplancton y el zooplancton, el cual es llevado a la vía detrítica, más que a la de los pastoreadores herbívoros (Cuadro 5). La ineficiencia en los sistemas de surgencia ha sido considerada por autores como Margalef (1985), quien menciona que más del $30 \%$ de la producción no es reciclada y es acumulada en el sedimento; esto es consistente con lo que se encontró en este estudio. En lo referente a la pradera de pastos marinos, Rodríguez-Ramírez y Garzón-Ferreira (2003) también encontraron para la bahía de Chengue (Parque Nacional Natural Tayrona), una ausencia generalizada de los potenciales consumidores directos de T. testudinium, como tortugas y manatíes. Sólo existen algunos herbívoros como Lytechinus variegatus, Tripneustes sp. y Sparisoma radians, que podrían estar aprovechando el recurso. Montoya-Maya (2002) encontró una situación similar en la composición de las especies que habitan la pradera de La Guajira y resaltó el hecho de una ausencia de pastoreo por parte de los grandes herbívoros como las tortugas. Diversos autores han discutido sobre la baja eficiencia en el pastoreo de los herbívoros como scáridos y equinodermos. 


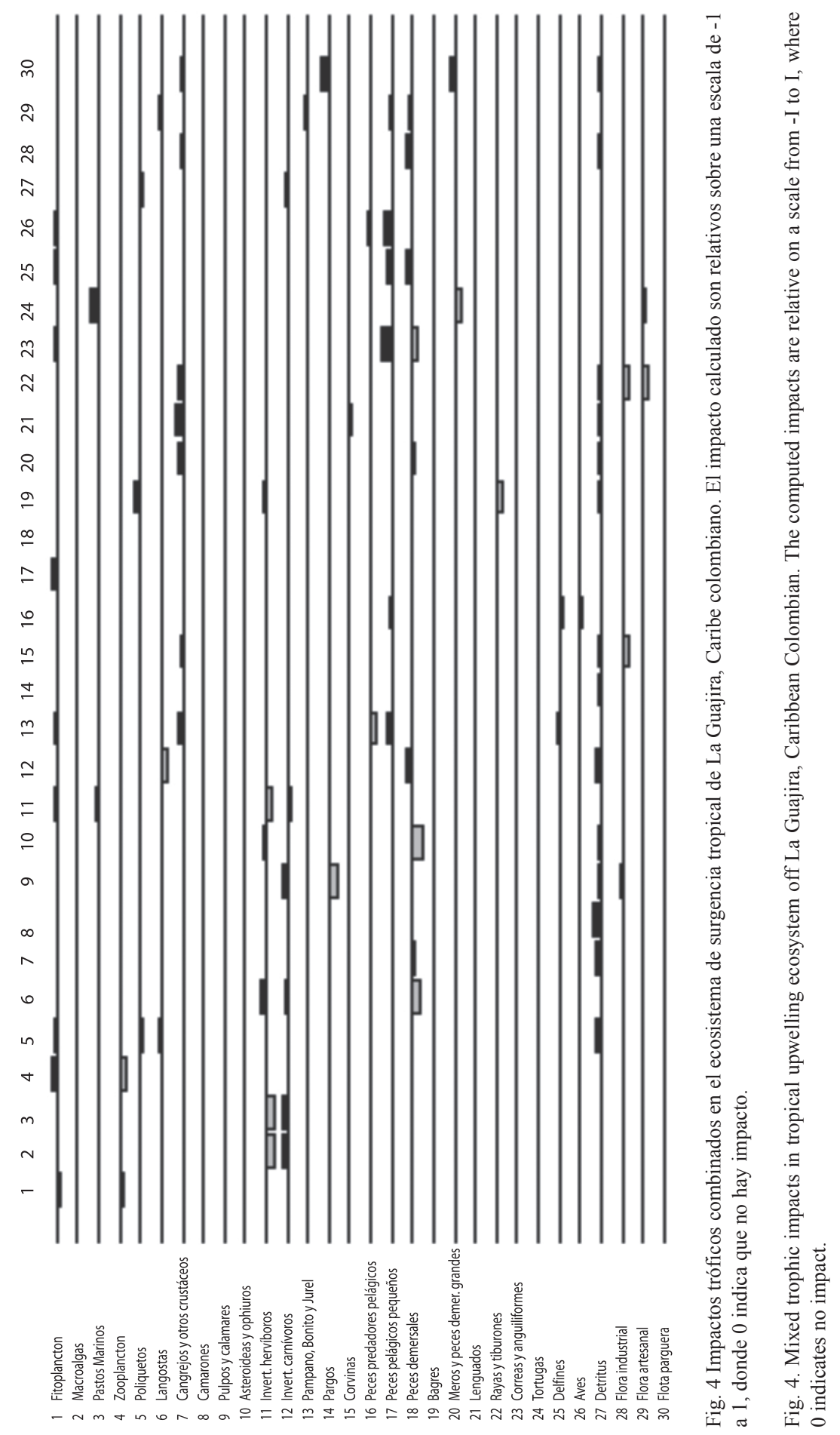



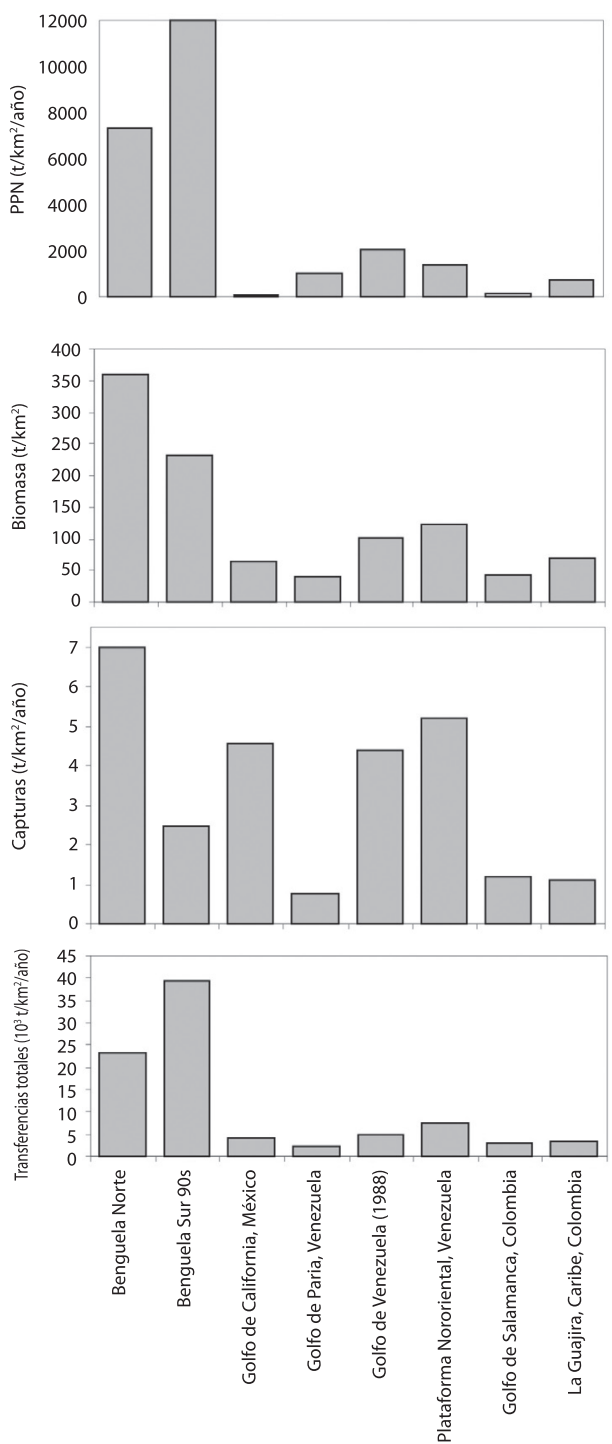

Fig. 5 Resumen estadístico comparativo de los modelos de áreas de surgencia. Se presentan las tendencias en todas de cuatro parámetros: Producción Primaria Neta, Biomasa total (excluyendo detritus), capturas totales y transferencias totales.

Fig. 5. Summary statistics comparative of upwelling balanced models. Trends in all four parameters: primary production, total biomass (excluding detritus), total catches and total system throughput.

Así, Van Rooij et al. (1998) encontraron que en los arrecifes de coral los scáridos consumen sólo el $22 \%$ de la biomasa algal. Greenway (1995) hizo una revisión de las relaciones tróficas en las praderas de Thalassia y encontró que sólo cuando existen densidades altas de equinodermos como Lytechinus, puede ocurrir una defoliación en la pradera; de lo contrario predomina la vía detrítica. Estas circunstancias generan un excedente en la producción primaria que finalmente es llevada al sedimento o exportada fuera del sistema, como lo menciona Lorenzen (1976).

También se encontró una alta densidad de pequeños pelágicos, lo cual es una característica propia de ecosistemas productivos, particularmente en las regiones de surgencia. La producción de estos grupos está representada por unas pocas especies (Bakun 1996). Pero lo más importante de este grupo en estos sistemas es que frecuentemente contribuyen notoriamente en las pesquerías mundiales (Hall 1999). En La Guajira, los pequeños pelágicos ocupan un nivel trófico (NT) intermedio (2.74), que es consistente con el nivel trófico encontrado en otros sitios de surgencia del Caribe colombiano (NT=2.6) (Duarte y García 2004). Pauly y Chistensen (1995) también encontraron un nivel trófico intermedio para este grupo en zonas de surgencia $(\mathrm{NT}=2.6)$, sugiriendo que tiene una mayor relación con el plancton que la que se puede dar en otros ecosistemas donde el nivel trófico que ocupan es superior a 3.0. En el ecosistema son depredados por aves, delfines, correas y anguiliformes, pámpano, bonito y jurel, y peces depredadores pelágicos, y como en otros ecosistemas de surgencia, la biomasa que remueven estos depredadores es más importante que la que se remueve por pesca (Cury et al. 2000). Los pequeños pelágicos de este sistema guardan similitud en las densidades de biomasa con otros sistemas de surgencia tropical. Sin embargo, la actividad de pesca de este recurso está limitada a la costa (Espeleta 1993). Los resultados indican que de la biomasa producida se utiliza el $1.25 \%$ en pesca y $31 \%$ en depredación; de manera similar Manjarrés et al. (1997) encontraron en las estimaciones de biomasa a través de la prospección hidroacústica que la proporción explotada de estos recursos es mínima en comparación con las biomasas estimadas. Los 
resultados sugieren que se debe hacer un estudio específico mediante simulación que lleve a una evaluación más detallada de este recurso y el efecto en el ecosistema que un incremento del esfuerzo pesquero, puede tener. Se debe considerar además que este recurso permanece todo el año (Páramo y Viaña 2002) y podría permitir la implementación de una nueva pesquería orientada hacia este recurso. No se debe olvidar que otros recursos explotados en el área como son los pargos, corvinas, rayas y tiburones están siendo sometidos a una explotación que de acuerdo al modelo, pone en peligro su capacidad de renovación como lo sugiere el hecho de que la mortalidad por pesca sea mayor que la mortalidad por depredación. Jarre y Christensen (1998) discutieron en torno a los resultados de mortalidad que estima Ecopath y encontraron que cuando la mortalidad por pesca sobrepaso a la mortalidad por depredación en la anchoveta peruana, sobrevino el colapso de la pesquería en la década de los 70 . De manera similar, Shannon et al. (2003) encontraron en el Sur de Benguela que para la anchoveta europea se incrementó la mortalidad por pesca en la década de 1990, sobrepasando a la mortalidad natural, sobreviniendo competencia entre depredadores naturales y la pesca por el recurso, lo cual ocasionó una disminución del mismo.

Los resultados indican que el $51 \%$ de lo que se pesca corresponde al nivel trófico III. Ecológicamente la explotación de peces de los niveles más altos es más costosa que la captura de invertebrados y pequeños pelágicos que generalmente ocupan niveles tróficos inferiores, puesto que la energía que entra a un nivel trófico es transferida en el siguiente nivel y la eficiencia en la transferencia trófica disminuye gradualmente hacia los niveles tróficos superiores debido al incremento en la tasa de respiración (Christensen y Pauly 1993). Como resultado la eficiencia en el rendimiento bruto de la pesquería que es de $0.001 \mathrm{t} / \mathrm{km}^{2} / \mathrm{año}$, guarda similitud con los rendimientos en otros ecosistemas del mundo que dirigen la pesca hacia los depredadores superiores (ArreguínSánchez 1993b, Manickchand-Heileman et al. 2004, Pomares 2001).
En sistemas naturales diversos se ha demostrado que las redes alimentarias presentan múltiples conexiones y los organismos toman el alimento de diferentes niveles tróficos, de acuerdo con la disponibilidad que halla en el medio, lo que los convierte en individuos oportunistas generalistas (Polis y Strong 1996). En el ecosistema de La Guajira se observa de forma similar un sobrelapamiento de las dietas en muchas de las especies; como resultado el Índice de Omnivoría es alto (3.2), aunque no se debe desconocer que la agregación de gran número de las especies en los grupos funcionales se relaciona con este resultado, especialmente en los grupos funcionales del nivel III, donde los grupos se encuentran próximos unos de otros (Fig. 2), y muchas de las especies comparten un amplio espectro dietario. Encontraron que la mayoría de los peces tropicales son oportunistas tróficos y presentan un espectro alimentario amplio que les permite adaptarse rápidamente a la disponibilidad de un recurso que esté abundante. Este grado de eurifagia en las especies tropicales se debe a la gran variedad faunística, acompañada de una relativamente baja biomasa de cada especie y trae como consecuencia un mayor gasto de energía en la búsqueda de alimento, en detrimento de conversión en biomasa.

Ahora bien, en sistemas de surgencia las condiciones del hábitat son variables a lo largo del año, lo que trae como consecuencia que los organismos no exploten de forma eficiente los recursos cuando estos son abundantes (Jarre-Teichman et al. 1998). Esto en términos de energía se traduce en que las transferencias de los productores primarios están alrededor del $10 \%$ en zonas de surgencia (Pauly y Christensen 1995). Cercano a este valor fue lo encontrado en La Guajira, que utiliza el $13 \%$ de la producción primaria para las transferencias de energía hacia los niveles tróficos superiores, y el $17 \%$ se pierde a la vía detrítica. Los resultados de la agregación trófica también muestran que los flujos desde los productores primarios son comparativamente menores que desde el detritus, señalando la importancia demersal en este ecosistema; se observa que el $32 \%$ de los 
flujos que se originan del detritus son destinados al consumo.

La relación entre la producción y la respiración resulta útil para establecer la medida de madurez en un ecosistema (Odum 1969). Al disminuir el valor de esta relación el sistema se vuelve más maduro. Odum consideraba a los sistemas de surgencia ecosistemas inmaduros, puesto que la producción excede en más de una unidad a la respiración total. Christensen y Pauly (1993) elaboraron un cuadro comparativo para ecosistemas acuáticos, y el rango fue de 0.8 a 3.2. Según lo anterior, el ecosistema de La Guajira es un sistema inmaduro, puesto que la relación entre producción primaria total y respiración total del sistema es 2.55. Otro de los atributos que se ha asumido como una medida de madurez es el ciclaje y longitud de las rutas, los cuales están correlacionadas con la capacidad de funcionamiento (estabilidad), esta relación se ha encontrado que es parabólica y sugiere una disminución de la estabilidad en la medida en que los valores en los índices de ciclaje son más altos; de esta manera se interpreta que un bajo ciclaje (sistemas de surgencia) es muy dependiente en energía, la cual recorre rápidamente el sistema y como tal es bastante inestable y vulnerable a los cambios en entrada nutrientes (Christensen y Pauly 1993). En la mayoría de los ecosistemas la longitud media de las vías se encuentra entre 2 y 4 según Finn (1976). En La Guajira este valor fue de 2.62 que, de acuerdo con lo señalado por Christensen y Pauly (1993), corresponde a vías cortas, como las que se presentan en los sistemas de surgencia y al mismo tiempo se relaciona con ecosistemas inmaduros, inestables sometidos a una constante perturbación en las condiciones del ecosistema.

Los atributos referidos al desarrollo del ecosistema que el Ecopath cuantifica son ascendencia, capacidad de desarrollo y estabilidad y han sido considerados para comparaciones entre diferentes ecosistemas (Christensen 1994, Perez-España y Arreguín-Sánchez 1999, Shannon et al. 2003). En este estudio la ascendencia relativa fue de $35.7 \%$, este valor es mayor que los encontrados en las dos últimas décadas en el sur de Benguela, los cuales fueron entre el $20 \%$ y $21 \%$ (Shannon et al. 2003). Por otro lado, en los modelos de surgencia producidos por Jarre-Teichmann (1998) los valores de ascendencia relativa para el Perú varían entre el $30 \%$ y $37 \%$, por lo cual podríamos decir que los valores encontrados en La Guajira están entre los valores encontrados en Perú. Luego la ascendencia en La Guajira presenta valores comparables al de un ecosistema de surgencia. La Guajira es un sistema de surgencia con una estabilidad comparable a la de sistemas sometidos a una alta variabilidad y en los que se deben incluir las surgencias tropicales como la del Nororiente de Venezuela, el Golfo de Venezuela y el Golfo de Salamanca (Mendoza 1993, Duarte y García 2004). Estos sistemas se encuentran influenciados por una surgencia costera ocasionada por los vientos alisios y presentan valores relativamente bajos en la ascendencia, entre $25.7 \%$ y $39.6 \%$ (Mendoza 1993, Pomares 2001, Duarte com. per.). En sistemas maduros como arrecifes de coral la ascendencia puede llegar hasta $50.4 \%$ (Pauly et al. 1993). Podemos decir que en sistemas de surgencia el valor en la ascendencia es relativamente bajo y que se relaciona con sistemas inestables poco desarrollados, de acuerdo con los atributos del ecosistema descritos por Odum (1969)

Los flujos de materia y energía en términos de tamaño en $\mathrm{t} / \mathrm{km}^{2} /$ año son mayores en los sistemas de grandes surgencias, como lo son el Norte y Sur de Benguela. Es claro que La Guajira presenta una surgencia moderada comparada con sistemas como Perú o Benguela con valores en las transferencias totales son superiores a $20000 \mathrm{t} / \mathrm{km}^{2} /$ año (Jarre-Teichman 1998, Shannon et al. 2003), el doble de lo que se puede llegar a presentar en surgencias tropicales donde estos valores no exceden 8000 t/ $\mathrm{km}^{2} / a n ̃ o$, como en el Golfo de Paria, Nor-oriente de Venezuela, Golfo de Salamanca y Golfo de Venezuela (Mendoza 1993, Duarte y García 2004, Pomares 2001, ManickchandHeileman et al. 2004, Fig. 4). Con respecto a la producción primaria y la biomasa, La Guajira presenta valores intermedios respecto a otros ecosistemas donde se presentan surgencias 
tropicales como la plataforma Nororiental de Venezuela y el Golfo de Venezuela. Las capturas en La Guajira difieren de otros sistemas de surgencia que tradicionalmente sustentan una fuerte producción pesquera basada en la extracción de pequeños pelágicos, la cual ha llegado a ser hasta el $10 \%$ de la captura mundial (Caddy y Sharp 1988). En La Guajira, aunque las biomasas de pequeños pelágicos guardan correspondencia con sitios de alta productividad, no se desarrolla una pesquería activa de este recurso. La extracción en este caso está dirigida a otros recursos, como camarones, pargos, meros y tiburones.

El nivel trófico de la pesquería en $\mathrm{La}$ Guajira varía entre 2.9 y 3.7 para las diferentes flotas, en estos niveles tróficos se encuentra gran parte de los peces depredadores del ecosistema. Los resultados indican que el $51 \%$ de lo que se pesca corresponde al nivel trófico III. Ecológicamente la explotación de peces de los niveles más altos es más costosa que la captura de invertebrados y pequeños pelági$\cos$ que generalmente ocupan niveles tróficos inferiores, puesto que la energía que entra a un nivel trófico es transferida al siguiente nivel y la eficiencia en la transferencia trófica disminuye gradualmente hacia los niveles tróficos superiores debido al incremento en la tasa de respiración (Christensen y Pauly 1993). Como resultado la eficiencia en el rendimiento bruto de la pesquería es $0.001 \mathrm{t} / \mathrm{km}^{2} / a n ̃ o$, guarda similitud con los rendimientos en otros ecosistemas del mundo que dirigen la pesca hacia los depredadores superiores (Arreguín-Sánchez 1993b, Manickchand-Heileman y Phillip 1996, Pomares 2001).

\section{AGRADECIMIENTOS}

MICH agradece al ZMT (Center for Tropical Marine Ecology) de la Universidad de Bremen -Alemania, por su cooperación logística y científica en el análisis del modelo $\mathrm{y}$ al personal del proyecto en el suministro de los insumos para el modelo. Esta investigación fue posible gracias al apoyo del Proyecto
“Dinámica espacio-temporal del ecosistema de afloramiento del área de Bocas de Ceniza-Punta Espada (Caribe colombiano) y sus implicaciones para un régimen de pesca responsable" (Universidad del Magdalena. COLCIENCIASINPA-INCODER-Universidad Nacional) cod: 3135-09-550-98.

\section{RESUMEN}

La Guajira es un ecosistema de surgencia explotado en la costa del Caribe colombiano. Con el propósito de integrar la información disponible sobre el sistema se construyó un modelo trófico de 27 grupos funcionales usando el programa Ecopath 5.0 Beta. Se obtuvo una base cuantitativa para compararlo con otros sistemas de surgencia. La Guajira presenta una biomasa total de 68 $\mathrm{t} / \mathrm{km}^{2} / \mathrm{año}$, la producción primaria neta del sistema es de $1248.51 \mathrm{t} / \mathrm{km}^{2} /$ año y las transferencias totales del sistema son de $3275 \mathrm{t} / \mathrm{km}^{2} /$ año, lo que indica un ecosistema con una surgencia moderada comparada con otros sitios. Las mayores transferencias de energía se dan del nivel trófico I-II $(68.93 \%)$, aunque existe una importante proporción de flujos al detritus (32\%). Los resultados en los atributos de madurez del sistema sugieren que La Guajira es un ecosistema inmaduro debido a que la relación $\mathrm{P} / \mathrm{R}$ excede a 1 y está en desarrollo debido a la baja ascendencia (33.7\%) y alta capacidad de desarrollo $(66.3 \%)$ como ocurre con otras surgencias donde se presentan valores de ascendencia entre $20 \%$ y $35 \%$. Aunque los datos básicos de entrada en el modelo fueron buenos, gracias a las investigaciones que se han elaborado entre 1995 y 2000 , existen algunos grupos donde no se encuentra aún información adecuada; especialmente en los valores de biomasa del fitoplancton, invertebrados, bagres, peces depredadores pelágicos, así como estimaciones de producción en invertebrados, peces depredadores pelágicos y peces pequeños pelágicos. No existe una cuantificación de las poblaciones de mamíferos y aves en el área de estudio, los cuales constituyen depredadores superiores y hacen parte esencial en sistemas de surgencia.

Palabras clave: relaciones tróficas, sistemas de surgencia, Ecopath, Colombia, Mar Caribe.

\section{REFERENCIAS}

Aguilera, L. 1985. Análisis del contenido estomacal y hábitos alimenticios del maro ojón Tylosurus acus (Lacepede, 1803) en la bahía de Mochima, estado 
Sucre. Tesis de Licenciatura, Universidad de Oriente, Cumaná, Venezuela. 28 p.

Andrade, C.A. 2000. The circulation and variability of the Colombian basin in the Caribbean sea. Dr. Thesis, University of Wales, Alberta, Canadá. 223 p.

Arreguín-Sánchez, F., J.C. Seijo \& E. Valero-Pacheco. 1993a. An application of Ecopath II to the North Continental shelf ecosystem of Yucatan, Mexico, p. 269-278. In V. Christensen \& D. Pauly (eds.). Trophic models of aquatic ecosystems. ICLARM. Conf. Proc. 26, Ciudad de Makati, Filipinas.

Arreguín-Sánchez, F., J.L. Munro, M.C. Balgos \& D. Pauly. 1993b. Biology, fisheries and culture of tropical groupers and snappers, p. 19-27. ICLARM conf. Proc. 48, Ciudad de Makati, Filipinas.

Avila, J.M. 1995. Aspectos biológicos y etológicos de delfines costeros con énfasis en la especie Sotalia fluviatilis (Delphinidae) en la bahía Cispata, Caribe colombiano. Tesis de Licenciatura, Universidad Jorge Tadeo Lozano, Bogotá, Colombia. 369 p.

Badaracco, M.T. \& R. Molinet. 1991. Flujos de materia en el sistema demerso-pelágico de Golfo Triste, Edo Carabobo, Venezuela. Tesis de Biología, Universidad Simon Bolivar, Caracas, Venezuela. 191 p.

Bakun, A. 1996. Patterns in the ocean. Ocean Processes and Marine Population Dynamics. California Sea Grant College System, National Oceanic and Atmospheric Administration, in cooperation with Centro de Investigaciones Biológicas del Noroeste, La Paz, México. 323 p.

Bedoya, J., O. Delgadillo \& P. Florez. 2000. Aportes a la caracterización de los desembarcos artesanales de pulpos, en Santa Marta y áreas adyacentes (Caribe colombiano), 10 p. Informe de Seminario de Investigación, Universidad Jorge Tadeo Lozano, Santa Marta, Colombia.

Bernal, A.R. 2000. Die struktur der zooplanktongemeinschaft im neritischen Bereich des Kolumbianischen Karibischen Meeres. InauguralDissertation Zur Erlangung des Doktorgrades der Naturwissenschaftlichen Fakultät der Justus-LiebigUnivesität Gießen, Alemania. 142 p.

Bitter, R. 1984. Composición de la dieta de Astropecten marginatus (Echinodermata: Asteroidea). Bol Inst. Oceanografía Venezuela. Univ. Oriente 23: 169-176.

Brulé, T., D.O. Ávila, M.S. Crespo \& C. Déniel. 1994. Seasonal and diel changes in diet composition of juvenile red grouper Epinephelus morio from Campeche Bank. Bull. Mar. Sci. 55: 255-262.

Cabrera, E. \& M.C. Donoso. 1993. Estudio de las características oceanográficas del Caribe colombiano Región III, Zona 1, PDCTM. Bol Cient. CIOH 13: 19-32.
Caddy, J.E. \& G.D. Sharp. 1988. Un marco ecológico para la investigación pesquera. FAO Docu. Tec. Pesca: $155 \mathrm{p}$.

Camacho, L.M. \& O.D. Galvis. 1980. Bahía Concha, aportes ecológicos: Parque Nacional Tayrona, Magdalena. Tesis de Licenciatura, Universidad Jorge Tadeo Lozano, Bogotá, Colombia. 179 p.

Campos, J.A., A. Segura, O. Lizano \& E. Madrigal. 1993. Ecología básica de Coryphaena hippurus (Pisces: Coryphaenidae) y abundancia de otros grandes pelágicos en el Pacífico de Costa Rica. Rev. Biol. Trop. 41:783-790.

Carles, C. 1971. Características biológico-pesqueras del bonito (Katsuwonus pelamis) y la albacora (Thunnus atlanticus) en la costa nororiental de Cuba. Inst. Nac. Pesca Cuba, Cent. Invest. Pesq., Contrib. 32, Cuba. $51 \mathrm{p}$.

Christensen, V. 1994. Emergy-based ascendency. Ecol. Model. 72: 129-144.

Christensen, V. \& D. Pauly. 1992. ECOPATH II - a software for balancing steady-state ecosystem models and calculating network characteristics. Ecol. Model. 61: 169-185.

Christensen, V. \& D. Pauly (eds.). 1993. Flow characteristics of aquatic ecosystems, p. 338-352. In V. Christensen \& D. Pauly (eds.). Trophic models of aquatic ecosystems. ICLARM Conf. Proc. 26, Makati, Filipinas.

Christensen, V., C.J. Walters \& D. Pauly. 2000. Ecopath with Ecosim Version 4, Help system C. ICLARM Software 6. Makati, Filipinas.

Corredor, J.E. 1979. Phytoplankton response to low level nutrient enrichment through upwelling in Columbian Caribbean Basin. Deep-Sea Research 26A: 731-741.

Correa, F. 1996. Proyecto investigación de los recursos bentónicos en La Guajira. Explotación y mortalidad de la Langosta en La Guajira, 18 p. Informe final Instituto Nacional de Pesca y Acuicultura, sector Guajira, Colombia.

Correa, F. \& L.M. Manjarrés. 2004. Inventario y caracterización general de las unidades económicas de pescas artesanales de la guajira, Caribe colombiano, p. 77-93. In L. Manjarrés. Pesquerías demersales del área norte del Caribe colombiano y parámetros ecológicos, biológico pesqueros y poblacionales del recurso pargo. Universidad del Magdalena, Santa Marta, Colombia.

Cortés, E. 1999. Standardized diet compositions and trophic levels of sharks. ICES J. Mar. Sci. 56: 707-717.

Cortés, M.L. \& M.M. Criales. 1990. Análisis del contenido estomacal del camarón Titi Xiphopenaeus kroyeri 
(Séller) (Crustacea: Natantia: Penaidea). An. Inst Invest. Mar y Costeras Punta de Betin 19-20: 23-33.

Crabtree, R.E., C.W. Harnden, D. Snodgrass \& C. Stevens. 1996. Age, growth, and mortality of bonefish, Albula vulpes, from the waters of the Florida keys. Fish. Bull 94: 442-451

Cury, P., A. Bakun, R. Crawford, A. Jarre, R. Quiñones, R. Shannon \& H. Verheye. 2000. Small pelagics in upwelling systems: patterns of interaction and structural changes in "wasp-waist" ecosystems. ICES J. Mar. Sci. 57: 603-618.

De La Cruz-Agüero, G. 1993. A preliminary model of Mandinga Lagoon, Veracruz, México, p. 193 - 197. In V. Christensen y D. Pauly. Trophic models of aquatic ecosystems. ICLARM. Conf. Proc. 26, Makati, Filipinas.

Díaz-Pulido, G. 2002. Vegetación marina de un sector de la plataforma continental de la Guajira (Caribe colombiano). Bol. Invest. Mar. Cost. 29: 27-34.

Díaz, J.M. \& M. Puyana. 1994. Moluscos del Caribe colombiano. Un catálogo ilustrado. COLCIENCIASFUNDACIÓN NATURA-INVEMAR. Presencia, Bogotá, Colombia. 291 p.

Díaz, J.M., L.M. Barrios \& D.I. Gómez. 2003. Las praderas de pastos marinos en el Caribe colombiano: Distribución y estructura de un ecosistema estratégico. INVEMAR, Publicación Especial No. 10. Santa Marta, Colombia. 140 p.

Domínguez, C. \& P.M. Alcolado. 1990. Características del macrofitobentos de la macrolaguna del Golfo de Batabano, p 8-17. In P.M. Alcolado (ed.). El bentos de la macrolaguna del Golfo de Batabano. Academia, La Habana, Cuba.

Duarte, LO, C.B. García, I. Moreno, G. Melo, P. Navajas, N. Sandoval \& D.von Schiller. 1999a. Atlas demográfico de los peces demersales del Golfo de Salamanca, Caribe colombiano. Dinámica poblacional, distribución alimentación y reproducción. Libro digital. CDROM. Invemar, Colciencias. ISBN 95950-6-5. Santa Marta, Colombia.

Duarte, L.O. \& C.B. García. 2004. Trophic role of small pelagic fishes in a tropical upwelling ecosystem. Ecol. Model. 172: 323-338.

Duarte, L.O., C.B. García, N. Sandoval, D. von Schiller, D. Melo G. \& P. Navajas. 1999b. Length-weight relationships of demersal fishes from the Gulf of Salamanca, Colombia. Naga, ICLARM Q. 22: 34-36.

Duque, G. 1993. Ecología trófica y aspectos reproductivos de las especies del género Oligoplites (Pisces: Carangidae) de la Ciénaga Grande de Santa Marta, Caribe colombiano. Tesis de Licenciatura. Universidad del Valle, Cali, Colombia. 100 p.
Elorduy, J.F. \& A.K. Peláez. 1996. Feeding habits of Cauloltilus affinis (Perciformes: Branchiostegidae) La Paz Bay, Baja California Sur, México. Rev. Biol. Trop. 44: 241-250.

Escorcia, F. \& F.J. Cuello. 1999. Capturas anuales, crecimiento, mortalidad y tasa de explotación del camarón rojo en el Caribe colombiano. Inpa, Cartagena, Colombia.

Espeleta, A. 1993. El machuelo (Ophisthonema oglinum) (Atlantic threat herring) y sus posibilidades de explotación en Colombia, 179-184 p. In Informe Técnico Final del Proyecto Integral de Investigaciones y Desarrollo de la Pesca Artesanal en el área Marítima de Santa Marta. INPA-CIID-UNIMAGDALENA, Santa Marta, Colombia.

Estrada, M.I. 1983. Contribución al conocimiento de la biología y ecología de las especies del género Haemulon (Pisces: Haemulidae) en los arrecifes del Caribe colombiano, con énfasis en la región de Santa Marta. Tesis de Licenciatura, Universidad Jorge Tadeo Lozano, Bogotá, Colombia. 129 p.

Finn, J.T. 1987. Measures of the ecosystem structure and function derived from analysis of flows. J. Theor. Biol. 56: 363-380.

Franco, L.E. 1996. Alimentación y reproducción de la lisa Mugil curema Valenciennes, 1836 (Pisces: Mugilidae) del Golfo de Cariaco, estado Sucre, Venezuela. Tesis de Maestría, Universidad de Oriente. Cumaná, Venezuela. $107 \mathrm{p}$.

Franks, J.S, N.M. Garber \& J.S. Warren. 1996. Stomach contents of juvenile cobia, Rachycentron canadum, from the northern Gulf of Mexico. Fish. Bull. 94: 374-380.

García, C.B. \& L.O. Duarte. 2002. Consumption to Biomass (Q/B) Ratio and Estimates of Q/B-predictor Parameters for Caribbean Fishes. NAGA, ICLARM Q. 25: 19-31.

García-Abad, C., A. Yánez-Arancibia, P. Sánchez-Gil \& M.T. García. 1992. Distribución, reproducción y alimentación de Syacium gunteri Ginsburg (Pisces: Bothidae), en el Golfo de México. Rev. Biol. Trop. 39: 27-34.

Gonzáles, E. 1981. Estudio bioecológico de los estadíos juveniles de peces de la Ciénaga Grande de Santa Marta y zonas adyacentes. Tesis de Licenciatura, Universidad Nacional de Colombia, Bogotá, Colombia. 89 p.

Greenway, M. 1995. Trophic relationships of macrofauna within a jamaican seagrass meadow and the role of the echinoid Lithechinus variegatus (Lamarck). Bull Mar. Sci. 56: 719-736.

Guzmán-Alvis, A.I. \& O.D. Solano. 1997. Solano Estructura de la taxocenosis Annelida-Mollusca en la región de 
Mingueo, Guajira (Caribe, colombiano). Bol. Invest. Mar. Cost. 26: 35-52.

Hall, S.J. 1999. The effects of fishing on marine ecosystems and communities. Blackwell Londres, Inglaterra. $274 \mathrm{p}$.

Hood, P. \& A. Johnson. 2000. Age, growth, mortality, and reproduction of red porgy, Pagrus pagrus, from the eastern Gulf of Mexico Fish. Bull. 98: 723-735.

Humphreys, W.F. 1979. Production and respiration in animal populations. J. Anim. Ecol. 48: 427-453.

Jarre-Teichman, A. 1992. Steady-state modelling of the peruvian upwelling ecosystem. Ph. D. Dissertation, Universidad de Bremen, Bremen, Alemania. 138 p.

Jarre-Teichman, A. 1998. The potential role of mass balance models for the management of upwelling ecosystems. Ecol. App. 8: 593-603.

Jarre-Teichman, A. \& V. Christensen. 1998. Comparative modelling of trophic flows in four large upwelling ecosystems vs local effects, p. 423-443. In M.H. Durand, P. Cury, R. Medelsossohn, C. Roy, A. Bakun $\&$ D. Pauly (eds.). Global vs local changes in upwelling ecosystems. Proceedings of the First International CEOS Meeting, September 6-8, 1994, Monterrey, C.A. EEUU. ORSTOM, París, Francia.

Labropoulou, M., A. Machias \& N. Tsimenides. 1999. Habitat selection and diet of juvenile red porgy, Pagrus pagrus (Linnaeus, 1758). Fish. Bull. 97: 495-507.

LeBorgne, R. 1982. Zooplancton production in the Eastern tropical Atlantic Ocean: net growth efficiency and P: $\mathrm{B}$ in terms of carbón, nitrogen and phosphorus. Lim. Ocean. 27: 681-698.

Lorenzen, C.J. 1976. Primary production in the sea, p. 173-185. In D.H. Cushing \& J.J. Walsh. (eds.). The ecology of the seas. W.B. Saunders, Filadelfia, Pensilvania, EEUU.

MacPherson, E. 1983. Ecología trófica de peces en las costas de Namibia. I. Hábitats alimentarios. Res. Exp. Cient. Inv. Pesq. Barcelona, España. 11: 81-137.

Manickchand-Heileman, S.C. \& D.A.T. Phillip. 1996. Reproduction, age and growth of the Caribbean red snapper Lutjanus purpureus in waters off Trinidad and Tobago. In R. Froese y D. Pauly Fishbase 2000 Concepts, design and data sources. ICLARM, Makati, Filipinas.

Manickchand-Heileman, S.C., J. Mendoza-Hill, A. Lum Kong \& F. Arocha. 2004. A trophic model for exploring possible ecosystem impacts of fishing in the Gulf of Paria, between Venezuela and Trinidad. Ecol. Model. 172: 307-322.
Manjarrés, L., G. Rodríguez, J. Torres, A. Vergara, J. Viaña, E. Arteaga, J. Arévalo, R. Galvis, J. Rodríguez, F. Amaya, D. García, J. Páramo \& G. De León. 1997. Crucero de evaluación de los peces pelágicos en el Caribe colombiano INPA-VECEP/INVEMAR/ DEMER 9703. Marzo de 1997, 23 p. In Informe final. Santa Marta, Colombia.

Manjarrés, L.M., J.C. Arévalo, J. Rodríguez \& P. Gómez. 2004a. Dinámica poblacional y manejo del stock de pargo rayado (Lutjanus synagris) (Linneaus, 1758) de La Guajira (Caribe colombiano), p. 267-296. In L. Manjarrés (ed). Pesquerías demersales del área norte del Caribe colombiano y parámetros ecológicos, biológico pesqueros y poblacionales del recurso pargo. Universidad del Magdalena, Santa Marta, Colombia.

Manjarrés, L.M., J.C. Arévalo, J. Rodríguez \& P. Gómez. 2004b. Mortalidad y estrategias de manejo del stock de Pargo palmero (Lutjanus analis) (cuvier, 1828) del área norte del Caribe colombiano, p. 297-317. In L. Manjarrés (ed). Pesquerías demersales del área norte del Caribe colombiano y parámetros ecológicos, biológico pesqueros y poblacionales del recurso pargo. Universidad del Magdalena, Santa Marta, Colombia.

Mann, K.H. 1982. Ecology of coastal waters. Stud. Ecol. 1: $1-322$.

Manooch III, C.S. \& W.T. Hogarth. 1981. Stomach contents and giant trematodes from wahoo, Acanthocybium solanderi, collected along the south Atlantic and coasts of the United States. Bull. Mar. Sci. 33: 227-238.

Manooch III, C.S. \& C. Barans. 1982. Distribution, abundance, age and growth of the Tomtate, Haemulon aurolineatum, along the southeastern United States coast. Fish. Bull. 80: 1-20.

Margalef, R. 1985. Primary production in upwelling areas. Energy, global ecology and resourses. In Simp. Int. Afl. O. Afr. Inst. Inv. Pesq. Barcelona. 1: 225-232.

Mendoza, J. 1993. A preliminary biomass budget for the northeastern Venezuela shelf ecosystem, p. 285-297. In V. Christensen \& D. Pauly (eds.). Trophic models of aquatic ecosystems. ICLARM. Conf. Proc. 26, Makati, Filipinas.

Montoya-Maya, P. 2002. Evaluación de la macrofauna epibentonica asociada a praderas de Thalassia testudinium (Banks ex König) en el Caribe colombiano. Tesis de Licenciatura, Universidad Jorge Tadeo Lozano, Bogotá, Colombia. 81 p.

Moreno, R. 1986. Ecología trófica de algunas especies de la familia Scombridae (Pisces) capturados en aguas costeras del departamento del Magdalena, Caribe colombiano. Tesis de Licenciatura, Universidad Nacional de Colombia, Bogotá, Colombia. 105 p. 
Murawski, S.A. 1991. Can we manage our multispecies. Fisheries 16: 5-13 p.

Muller-Karger, F.E. \& R. Aparicio. 1994. Mesoscale processes affecting phytoplankton abundance in the Southeastern Caribbean Sea. Cont. Shelf. Res. 14: 199-221.

Navas, G., L.S. Mejía, N. Ardila \& J. Reyes. 2002. Caracterización y catalogación de la macrofauna marina del Caribe colombiano, Fase 2, 14 p. +61 anexos. In Informe técnico final. Proyecto INVEMARCOLCIENCIAS. Instituto de Investigaciones Marinas y Costeras (INVEMAR), Santa Marta, Colombia.

Naviera, J.L. 1996. El orden Cetacea en la región nororiental de Venezuela. Tesis de Maestría, Universidad de Oriente, Cumaná, Venezuela. 229 p.

Obando, E. 1987. Aspectos biológicos y alimenticios del bolo Diplectrum formosum (Linnaeus, 1766) de la costa sureste de Margarita, Venezuela. Tesis de Maestría, Universidad de Oriente, Cumaná, Venezuela. 169 p.

Odum, E.P. 1969. The strategy of ecosystem development: an understanding of ecological succession provides a basis for resolving man's conflict with nature. Science 164: 262-270.

Odum, W.E. \& E.J. Heald 1975. The detritus based food web of an estuarine mangrove community, 265-286 p. In L.E. Cronin (ed.). Est. Research Vol 1. Academic, Nueva York, EEUU.

Ospina, J.F. \& F.I. Pardo. 1993. Evaluación del estado de madurez gonadal y los hábitos alimenticios de la ictiofauna presente en la Bahía de Cartagena. Tesis de Licenciatura, Universidad Jorge Tadeo Lozano, Bogotá, Colombia. 180 p.

O’Neill, R.V., D.L. DeAngelis, J.B. Waide \& T.F.H. Allen. 1986.A hierarchical concept of ecosystem. Princeton, Nueva Jersey, EEUU. 253 p.

Opitz, S. 1993. A quantitative model of the trophic interactions in a Caribbean reef ecosystem, p. 259-267. In V. Christensen \& D. Pauly (eds.). Trophic models of aquatic ecosystems. ICLARM. Conf. Proc. 26. Makati, Filipinas.

Opitz, S. 1996. Trophic interactions in Caribbean coral reefs. ICLARM. Tech Rep. 43, Makati, Filipinas. $341 \mathrm{p}$.

Páramo, J.E. \& J.E. Viaña. 2002. Evaluación hidroacústica del machuelo (Ophisthonema oglinum) y la sardina (Sardinella aurita), en la zona norte del Caribe colombiano, durante julio-agosto y diciembre de 1997. Bol. Inst. Invest. Mar y Costeras INVEMAR 31: 33-52.
Parra, B.J. 1984. Análisis del contenido estomacal y habitos alimenticios de la camiguana Anchoa parva (Meek y Hildebrande, 1923), (Pisces: Engraulidae) del Golfo de Cariaco, Edo. Sucre, Venezuela. Bol Inst. Oceanogr. Venezuela Univ. Oriente 23: 3-13.

Pauly, D. \& V. Christensen. 1995. Primary production required to sustain global fisheries. Nature 374: 255-257.

Pauly, D., V. Sambilay \& S. Opitz. 1993. Estimates of relative food consumption by fish and invertebrate population, required for modelling the Bolinao reef ecosystem, Philippines, p. 236-251. In V. Christensen $\&$ D. Pauly (eds.). Trophic models of aquatic ecosystems. ICLARM Conf. Proc. 26, Makati, Filipinas.

Penchaszadeh, P.E. \& M.E. Lera. 1983. Alimentación de tres especies tropicales de Luidia (Echinodermata, Asteroidea) en Golfo Triste, Venezuela. Carb. Journ Sc. 19: 1-2.

Pereira, C. 1995. Estimación del camarón rosado Penaeus (Farfantepenaeus) notialis (Perez-Farfante, 1967) en la zonas sur y norte del Caribe colombiano durante 1993. Tesis de Licenciatura, Universidad Jorge Tadeo Lozano. Bogotá, Colombia. 59 p.

Perez-España, H. \& F. Arreguín-Sánchez. 1999. A measure of ecosystem maturity. Ecol. Model. 119: 79-85.

Pinilla, G.A. 1986. Ecología trófica del jurel (Caranx hippos Linnaeus, 1766), la Cojinoa (Caranx crysos Mitchill, 1815) y el ojo gordo (Selar crumenophtalmus Bloch,1793) (Pisces: Carangidae), en aguas costeras del departamento del Magdalena, Caribe colombiano. Tesis de Licenciatura, Universidad de Colombia, Bogotá, Colombia. 90 p.

Pitts, P.A. 1991. Comparative use of food and space by three Bahamian butterflyfishes. Bull. Mar. Sci. 48: 749-756.

Polis, G.A. \& D.R. Strong. 1996. Food web complexity and community dynamics. Am. Nat.: 147: 813-846.

Polovina, J.J. 1984a. Model of a coral reef ecosystem I. The ECOPATH model and its application to french frigate shoals. Coral Reefs 3: 1-11.

Polovina, J.J. 1984b. An overview of the ECOPATH model. Fishbyte 2: 5-7.

Polovina, J.J. \& M.D. Ow. 1983. ECOPATH: a user's manual and program listings. Nat. Mar. Fish. Serv., NOAA, Honolulu Admin. Rep. H-83-23. 46 p.

Pomares, O. 2001. Estructura espacio-temporal y trófica de las comunidades acuáticas del Golfo de Venezuela. Tesis de grado, Universidad Central de Venezuela. Caracas, Venezuela. 162 p. 
Proyecto INPA-COLCIENCIAS. 2002. Evaluación de las pesquerías demersales del área norte del Caribe colombiano y parámetros ecológicos, biológico-pesqueros y poblacionales del recurso pargo INPACOLCIENCIAS cod. 3135-09-550-98. INPA, Santa Marta, Colombia.

Proyecto INVEMAR. 2001. Construcción del modelo trófico en la subárea sur de la zona nerítica del Pacífico colombiano utilizando el programa Ecopath con Ecosim. In Informe técnico. Buenaventura, Colombia.

Proyecto INVEMAR-COLCIENCIAS. 2002. Caracterización y catalogación de la macrofauna marina del Caribe colombiano, Fase 2, 14p. +61 anexos. In Informe técnico final. Instituto de Investigaciones Marinas y Costeras (INVEMAR), Santa Marta, Colombia.

Quintero, A.J. 2000. Composición de la comunidad íctica de la Bahía de Cispatá, Caribe colombiano, y hábitos alimentarios de las especies del género Mugil presentes en el área. Tesis de Licenciatura, Universidad Javeriana, Bogotá, Colombia. 158 p.

Randall, J.E. 1967. Food habits of reef fishes of the West Indies. Stud. Trop. Ocean. Miami 5: 665-847.

Reyes, A.A. 1999. Hábitos alimentarios y algunos aspectos reproductivos de la cojinoa negra Caranx crysos (Mitchill, 1815) capturada con chinchorro en la bahía de Taganga, Caribe colombiano Tesis de Licenciatura, Universidad Jorge Tadeo Lozano, Santa Marta, Colombia. 131 p.

Rincón, M.C., M.F. Huq \& I. Ramírez-Arredondo. 1988. Aspectos alimenticios de la sardina, Sardinella aurita Valenciennes, 1847 (Pises: Clupeidae) de los alrededores de la región de la Península de Araya y alrededores de la Islas de Coche y Cabagua, Venezuela. Bol. Inst. Oceanogr. Venezuela, Univ. Oriente 27:129-143.

Rodríguez-Ramírez, A. \& J. Garzón-Ferreira. 2003. Monitoreo de arrecifes coralinos, pastos marinos y manglares en la Bahía de Chengue (Caribe colombiano): 1993-1999. INVEMAR, Santa Marta, Serie Publicaciones Especiales 8: 170 p.

Schmidt, T.W. 1989. Food habits length-Weight relationship and condition factor of young great barracuda Syphraena barracuda (Walbaum), from Florida bay, Everglades National Park, Florida. Bull. Mar. Sci. 44: $163-170$.
Shannon, L.J., C.L. Moloney, A. Jarre \& J.G. Field. 2003. Trophic flows in the southern Benguela during the 1980s and 1990s. J. Mar. Syst. 39: 83-116.

Sedberry, G.R. \& N. Cuellar. 1993. Planktonic and benthic feeding by the reef-associated vermilion snapper, Rhomboplites aurorubens (Teleostei, Lutjanidae). Fish. Bull. 91: 699-709.

Tranter, D.J. 1976. Herbivore production, p. 186-224. In D.H. Cushing \& J.J. Walsh. The ecology of the seas. W.B. Saunders, Pensilvania, EEUU.

Ulanowicz, R.E. 1986. Growth and development. Ecosystem phenomenology. Springer, Berlín, Alemania. 204 p.

Valiela, I. 1995. Marine ecological processes. Springer, Nueva York, EEUU. 686 p.

Van Rooij, J.M., J.J. Videler \& J.H. Bruggemann. 1998. High biomass and production but low energy transfer efficiency of Caribbean parrotfish: implications for trophic models of coral reefs. J. Fish Biol. 53 (Supl. A): $154-178$.

Vergara, S. 1990. Vertebrados terrestres, p. 85-100. In M.C. Jimeno (ed.). Colombia Caribe. Fondo José Celestino Mutis -FEN, Bogotá, Colombia.

Viaña, J., A. Medina, M. Barros, L. Manjares, J. Altamar \& M. Solano. 2004. Evaluación de la ictiofauna demersal extraída por la pesquería industrial de arrastre en el área norte del caribe colombiano (enero/2000-junio/2001), p. 115-154. In L. Manjarrés (ed.). Pesquerías demersales del área norte del Caribe colombiano y parámetros ecológicos, biológico pesqueros y poblacionales del recurso pargo. Universidad del Magdalena, Santa Marta, Colombia.

Wolff, M. 1993. A trophic model for Tongoy Bay -a system exposed to suspended scallop (Northern Chile). J. Exp. Mar. Biol. Ecol. 182: 149-168.

Wolff, M., H.J. Hartmann \& V. Koch. 1996. A pilot trophic model for Golfo Dulce, a fjord-like tropical embayment, Costa Rica. Rev. Biol. Trop. 44 (Supl. 3): $215-231$

Wolff, M., V. Koch, J. Bautista \& J.A. Vargas. 1998. A trophic flow model of the Golfo the Nicoya, Costa Rica. Rev. Biol. Trop 46. (Supl. 6): 63-79.

Zimmerman, R.J. 1979. Seagrasses and the evolution of marine gammaridean amphipods. Proc. Assoc. Isl. Mar. Lab. Caribb. 17 p. 